"This document is the Accepted Manuscript version of a Published Work that appeared in final form in Journal of chemical \&engineering data, copyright (C) American Chemical Society after peer review and technical editing by the publisher. To access the final edited and published work see http://pubsdc3.acs.org/articlesonrequest/AORUCHbPm4Pwe6R2H7cFAut 


\title{
The Ideal Gas Thermochemistry of Oxonium Cations
}

\author{
Alexander Burcat* ${ }^{\dagger}$ Elke Goos ${ }^{\ddagger}$ \\ ${ }^{\dagger}$ Faculty of Aerospace Engineering, Technion-IIT, Haifa, 32000, Israel \\ ${ }^{\ddagger}$ Institute of Combustion Technology, DLR (German Aerospace Center), \\ Stuttgart, 70569 Germany.
}

* corresponding author. e-mail: aer0201@ technion.ac.il

\begin{abstract}
The ideal gas thermochemistry of twenty carbon oxonium cations were calculated for the first time using the DFT G3B3 method. The cations are $\mathrm{CH}-\mathrm{OH}_{2}+, \mathrm{CH}_{2}-\mathrm{OH}_{2}+, \mathrm{CH}_{3}-$ $\mathrm{OH}_{2}+, \mathrm{CH}_{3} \mathrm{O}=\mathrm{CH}_{2}+,\left[\mathrm{CH}_{2}-\mathrm{CH}_{2}\right] \mathrm{OH}+, \mathrm{CH}_{3} \mathrm{OH}-\mathrm{CH}_{2}+,\left(\mathrm{CH}_{3}\right)_{2} \mathrm{OH}+,\left(\mathrm{CH}_{3}\right)_{3} \mathrm{O}+, n-$ $\mathrm{C}_{3} \mathrm{H}_{7} \mathrm{OH}_{2}+,\left(\mathrm{CH}_{3}\right)_{2} \mathrm{CHOH}_{2}+,\left(\mathrm{CH}_{3}\right)_{2} \mathrm{OCH}_{2}+, \mathrm{CH}_{3}\left[\mathrm{CH}-\mathrm{CH}_{2}\right] \mathrm{OH}+,\left[\mathrm{CH}_{2}-\mathrm{CH}_{2}-\mathrm{CH}_{2}\right] \mathrm{OH}+$, $\left(\mathrm{CH}_{3}\right)_{2} \mathrm{OC}_{2} \mathrm{H}_{5}+,\left[\mathrm{CH}_{2}-\mathrm{CH}_{2}-\mathrm{CH}_{2}-\mathrm{CH}_{2}\right] \mathrm{OH}+, \mathrm{C}_{2} \mathrm{H}_{5} \mathrm{OH}_{2}+,\left(\mathrm{C}_{2} \mathrm{H}_{5}\right)_{2} \mathrm{OH}+,\left(\mathrm{C}_{2} \mathrm{H}_{5}\right)_{2} \mathrm{OCH}_{3}+$, $\left[\mathrm{CH}_{2}-\mathrm{CH}_{2}-\mathrm{CH}_{2}-\mathrm{CH}_{2}-\mathrm{CH}_{2}\right] \mathrm{OH}+,\left(\mathrm{C}_{2} \mathrm{H}_{5}\right)_{3} \mathrm{O}+$. The data are presented (in the Supporting Information) as 7 term NASA polynomials, to be used by chemical kineticists. Wherever available, estimates of the enthalpies of formation are compared to the literature.
\end{abstract}

\section{INTRODUCTION.}

In the last 20-30 years, much attention was given, both industrially and theoretically to production of methane from waste fermentation and its use, along with natural gas, to produce methanol and dimethylether over zeolite catalysts. These are further converted to higher olefin compounds.

It was proposed ${ }^{1,2}$ that these processes occur via different carbon Oxonium cations. Oxonium cations are defined as ions in which the Oxygen atoms are connected to three different atoms or in some cases the Oxygen is connected to two carbons only but with a double bond to one of them. In cases where the third atom is hydrogen the oxonium is also called a 'protonated molecule'. However the name Oxonium is not specific since sometimes the word oxonium is included by IUPAC in compounds like Methyloxoniumylidene $\mathrm{CH}_{3} \mathrm{O}+$ which does not satisfy the above definition. 
Lesthaeghe et al. $^{3}$ have calculated different paths for the acidic processes of methanol over catalysts while using a few carbon Oxonium cations.

To further perform kinetic reaction calculations by the Chemkin ${ }^{4}$ or Cantera ${ }^{5}$, kinetic programs, the thermochemistry of the included specie has to be provided in the polynomial form. However the thermochemistry of the simplest carbon oxonium cations is not known.

This article is devoted to the calculation of the thermochemical data of the following oxonium cations: $\mathrm{CH}-\mathrm{OH}_{2}+, \mathrm{CH}_{2}-\mathrm{OH}_{2}+, \mathrm{CH}_{3}-\mathrm{OH}_{2}+, \mathrm{CH}_{3} \mathrm{O}=\mathrm{CH}_{2}+,\left[\mathrm{CH}_{2}-\right.$ $\left.\mathrm{CH}_{2}\right] \mathrm{OH}+, \mathrm{CH}_{3} \mathrm{OH}-\mathrm{CH}_{2}+, \quad\left(\mathrm{CH}_{3}\right)_{2} \mathrm{OH}+, \quad\left(\mathrm{CH}_{3}\right)_{3} \mathrm{O}+, \quad n-\mathrm{C}_{3} \mathrm{H}_{7} \mathrm{OH}_{2}+, \quad\left(\mathrm{CH}_{3}\right)_{2} \mathrm{CHOH}_{2}+$, $\left(\mathrm{CH}_{3}\right)_{2} \mathrm{OCH}_{2}+, \mathrm{CH}_{3}\left[\mathrm{CH}-\mathrm{CH}_{2}\right] \mathrm{OH}+,\left[\mathrm{CH}_{2}-\mathrm{CH}_{2}-\mathrm{CH}_{2}\right] \mathrm{OH}+,\left(\mathrm{CH}_{3}\right)_{2} \mathrm{OC}_{2} \mathrm{H}_{5}+,\left[\mathrm{CH}_{2}-\mathrm{CH}_{2}-\right.$ $\left.\mathrm{CH}_{2}-\mathrm{CH}_{2}\right] \mathrm{OH}+, \quad \mathrm{C}_{2} \mathrm{H}_{5} \mathrm{OH}_{2}+, \quad\left(\mathrm{C}_{2} \mathrm{H}_{5}\right)_{2} \mathrm{OH}+, \quad\left(\mathrm{C}_{2} \mathrm{H}_{5}\right)_{2} \mathrm{OCH}_{3}+, \quad\left[\mathrm{CH}_{2}-\mathrm{CH}_{2}-\mathrm{CH}_{2}-\mathrm{CH}_{2}-\right.$ $\left.\mathrm{CH}_{2}\right] \mathrm{OH}+,\left(\mathrm{C}_{2} \mathrm{H}_{5}\right)_{3} \mathrm{O}+$

\section{CAlCulations.}

The thermochemistry of cations and anions cannot be calculated using Benson's ${ }^{6}$ group additivity method. The enthalpy of formation is normally estimated from different measurements involving ionization energies. Other thermochemical properties are usually not reported.

The enthalpies of formation of ions are expressed by two historically separate conventions. One is called the "thermal electron" convention (also known as the "electron convention"), used by thermodynamicists and the other the "stationary electron" convention (also known as "ion convention") used by chemists and physicists. The difference between the two conventions is the value of the enthalpy increment $H_{T}-H_{0}$ of the "electron gas". In the "thermal electron" convention, it is set to $2.5 R T$, while in the "stationary electron" convention the enthalpy increment is zero at all temperatures. Thus the enthalpies of formation and the Gibbs energies of formation of all ions, are different in the two conventions but not the other thermodynamic values, such as entropies.

Because this article deals with thermodynamics we have chosen the "thermal electron" convention, which is also the convention adopted by the JANAF Tables ${ }^{7}$, Gurvich Tables ${ }^{9}$ and the NBS Tables ${ }^{8}$.

Most ion chemists in their publications use the "stationary electron" convention without mentioning it, and also the Lias et al. ${ }^{10,19}$ compilation of ion thermochemistry nicknamed as the GIANT (and also the NIST WebBook, ${ }^{11}$ which uses Lias et al. values for ions). Using values from the two conventions in the same calculation results in serious errors. Therefore, it is essential that the values for all charged species be expressed within the same convention. 
It is simple to change the enthalpies of formation from one convention to the other. In both conventions at $0 \mathrm{~K}$, the enthalpies of formation are identical. At other temperatures, the value of the "stationary electron" can be converted to the "thermal electron" convention for the enthalpy of formation by adding the quantity $q \times 2.5 R T$, where $q$ is the charge of the ion and $2.5 R T=6.197 \mathrm{~kJ} \mathrm{~mol}^{-1}$ at $298.15 \mathrm{~K}$,

$$
\begin{aligned}
& \Delta_{f} H_{T}^{\text {thermal conv. }}\left(\mathrm{ABC}^{+q}\right)=\Delta_{f} H_{T}^{\text {stationary conv. }}\left(\mathrm{ABC}^{+q}\right)+q 2.5 R T \\
& \Delta_{f} H_{T}^{\text {thermal conv. }}\left(\mathrm{ABC}^{-q}\right)=\Delta_{f} H_{T}^{\text {stationary conv. }}\left(\mathrm{ABC}^{-q}\right)-q 2.5 R T
\end{aligned}
$$

Thus, $\Delta_{f} H 298\left(\mathrm{H}^{+}\right)=1530.047 \mathrm{~kJ} \mathrm{~mol}^{-1}$ under the "stationary electron" convention is $1536.244 \mathrm{~kJ} / \mathrm{mol}$, under the "thermal electron" convention, as used in this article. Also, $\Delta_{f} H 298\left(\mathrm{H}^{-}\right)=145.228 \mathrm{~kJ} \mathrm{~mol}^{-1}$ under the "stationary electron" convention becomes $139.031 \mathrm{~kJ} \mathrm{~mol}^{-1}$, under the "thermal electron" convention, by subtracting $6.197 \mathrm{~kJ} \mathrm{~mol}^{-1}$ (since the charge is -1 ).

Presently the $a b$-initio calculations provide good means for the calculation of the thermochemistry of ions, and specifically DFT calculations provide a fast and economic way for this task.

The oxonium cations were calculated with the G3//B3LYP (G3B3) method. Although there are many protocol methods for estimating the enthalpy of formation like Gn, Wn, CBS etc. we have preferred G3B3 because its estimates are within the same range of error as the others ${ }^{22,23}$ and also in order to be on the same calculation level as other ions radicals and molecules we calculated in our thermochemical database ${ }^{20}$. It should also be mentioned that the NIST Computational Chemistry Comparison Benchmark $(\mathrm{CCCBDB})^{21}$ site prefers the G3B3 method over others because more calculations are devoted to it than to other methods.

In order to facilitate the comparison of data between a given set of calculations the atomization method was chosen, because using isodesmic or isogyric reactions infers the use of different reactions for every species. Baboul et al. ${ }^{12}$ have defined the scheme for the G3//B3LYP calculations, and we used the Gaussian $03{ }^{13}$ program suite. The 
Chem $3 \mathrm{D}^{14}$ program was used to draw the species for the Gaussian input coordinates. The Gaussian optimized the geometry provided by the Chem $3 \mathrm{D}^{14}$ package and the vibration frequencies were computed at the $\mathrm{B} 3 \mathrm{LYP} / 6-31 \mathrm{G}(d)$ level. After that single point energies were computed at the QCISD(T,E4T)/6-31G(d), MP4/6-31+G(d), MP4/6-31G(2df,p), and MP2(Full)/G3Large levels. The Gaussian output was scanned by a custom-written script, which collects and computes all the relevant quantities, and displays the final enthalpy of formation of the calculated specie at $298 \mathrm{~K}$ in the "stationary electron convention.

The results of the G3B3 method ${ }^{12}$ are at the QCISD(T,Full)/G3Large level (atomization), assuming that the calculated contributions are additive.

To handle the problem of closed shell compounds (the protonated and other oxonium species) and the open shell compounds (the radical cations/distonic ions) it was necessary to specify in the Gaussian input if the species is a singlet or doublet.

The estimated uncertainty of the enthalpy of formation at $298 \mathrm{~K}$ is $8 \mathrm{~kJ} \mathrm{~mol}^{-1}$, which approximates the $95 \%(2 \sigma)$ confidence limit customary in thermochemistry. It is calculated as twice the value of the mean absolute error of the method which is $\approx 4 \mathrm{~kJ}$ $\mathrm{mol}^{-1}$.

The thermochemistry of the 20 Oxonium cations reported in this article were calculated with the Gordon and McBride NASA program ${ }^{15}$. The B3LYP/6-31G(d) not scaled vibrations from the Gaussian output were used (because they fit better the experimental IR values). For the NASA program we used the Gaussian calculated moments of inertia, the reduced moments of internal rotation as well as estimated rotation barrier energies. The enthalpy of formation at $298 \mathrm{~K}$ calculated by the G3B3 method and adjusted to the thermal electron convention, was also used. The program ${ }^{15}$ also performs the calculation of species polynomials.

The data used for the polynomial calculation are listed in Table 1. The thermochemical data at $\mathrm{T}=298.15 \mathrm{~K}$ are listed in Table 2 . The values given are from the data table calculated by the PAC program ${ }^{15}$. The polynomials are listed in Table S1 (in the supporting information). The polynomials are subject to inherent fitting errors caused by the 7 term limitations, therefore produce very slightly different values than given in Table 2 . 
Table 1 Data used to calculate the polynomials

1). CAS \# N/A

$\mathrm{CHOH}_{2}+$ Oxonium Methylidene $\sigma=1$ STATWT $=1$

$\mathrm{I}_{\mathrm{A}}=0.4111 \mathrm{I}_{\mathrm{B}}=2.8751 \mathrm{I}_{\mathrm{C}}=3.2862 \mathrm{~g} \mathrm{~cm}^{2} \times 10^{-39}$

$\nu=577,902,933,989,1438,1688,3029,3336,3545 \mathrm{~cm}^{-1} \quad \mathrm{REF}=\mathrm{B} 3 \mathrm{LYP} / 6-31 \mathrm{G}(\mathrm{d})$

$\Delta_{\boldsymbol{f}} \mathbf{H 2 9 8}=\mathbf{1 0 4 2 . 0 5} \pm \mathbf{8} . \mathbf{k J ~ m o l}^{-1}$ (thermal electron) $\mathrm{REF}=$ Burcat G3B3

2). CAS \# N/A

$\mathrm{CH}_{2} \mathrm{OH}_{2}{ }^{*}+$ Methylene Oxonium Radical $\sigma=1$ STATWT $=2$

$\mathrm{I}_{\mathrm{A}}=0.5896 \mathrm{I}_{\mathrm{B}}=3.3779 \mathrm{I}_{\mathrm{C}}=3.853 \mathrm{~g} \mathrm{~cm}^{2} \times 10^{-39}$

$v=278,683.5,717,934,935,1290.5,1434,1711,3200,3375,3551,3629 \mathrm{~cm}^{-1} \mathrm{REF}=\mathrm{G} 3 \mathrm{~B} 3$

$\Delta_{\boldsymbol{f}} \mathbf{H 2 9 8}=\mathbf{8 2 5 . 9} \pm \mathbf{4 . 5} \mathbf{~ k J ~ m o l}^{-1}$ (thermal electron) REF=Ruscic ATcT $2013^{16}$

$\left\{\Delta_{f} H 298=826.7 \pm 8 \mathrm{~kJ} \mathrm{~mol}^{-1}\right.$ (thermal electron) $\left.\mathrm{REF}=\mathrm{Burcat} \mathrm{G} 3 \mathrm{~B} 3\right\}$

$\left\{\left\{\Delta_{f} H 298=815 \pm 8 \mathrm{~kJ} \mathrm{~mol}^{-1}\right.\right.$ (thermal electron) $\mathrm{REF}=$ Lias et al. $\left.{ }^{10,19}\right\}$

3). CAS \# 323194-33-8

$\mathrm{CH}_{5} \mathrm{O}+\mathrm{CH}_{3}-\mathrm{OH}_{2}+$ Protonated Methanol cation (Methyl Oxonium) Rydberg cation

$\sigma=1 \quad$ STATWT $=1$

$\mathrm{I}_{\mathrm{A}}=0.8108 \mathrm{I}_{\mathrm{B}}=4.0126 \mathrm{I}_{\mathrm{C}}=4.1771 \mathrm{~g} \mathrm{~cm}^{2} \times 10^{-39}$

$v=251,727,812,941,1169,1290,1475.5(2), 1496,1713,3124,3250,3258,3586,3669 \mathrm{~cm}^{-1}$

$\mathrm{REF}=\mathrm{G} 3 \mathrm{~B} 3$ the values are $\pm 2 \mathrm{~cm}^{-1}$ of the values calculated by NIST ${ }^{18}$

$\Delta_{f} \mathbf{H 2 9 8}=\mathbf{5 8 3 . 7} \pm \mathbf{8} \mathbf{~ k J ~ m o l}^{-1}$ REF=Burcat G3B3 (thermal electron)

$\left\{\Delta_{f} H 298=592 . \mathrm{kJ} \mathrm{mol}^{-1} \mathrm{REF}=\right.$ Brown \& Eraslan estim. ${ }^{17}$ (thermal electron) $\}$

$\left\{\Delta_{f} H 298=573 . \mathrm{kJ} \mathrm{mol}^{-1} \mathrm{REF}=\right.$ Lias et al. ${ }^{10,19}$ (thermal electron) $\}$ 
4). CAS \# 23653-97-6

$\mathrm{C}_{2} \mathrm{H}_{5} \mathrm{O}+\mathrm{CH}_{3} \mathrm{O}=\mathrm{CH}_{2}+$ Oxonium MethylMethylene $\sigma=1$ STATWT $=1$

$\mathrm{I}_{\mathrm{A}}=1.5384 \quad \mathrm{I}_{\mathrm{B}}=8.2382 \quad \mathrm{I}_{\mathrm{C}}=9.2349 \mathrm{~g} \mathrm{~cm}^{2} \times 10^{-39}$

$v=167,435,582,790,1165,1180,1207,1284,1447,1471,1497,1514,1714,3100,3113,3224$,

$3227,3256 \mathrm{~cm}^{-1} \quad \mathrm{REF}=\mathrm{G} 3 \mathrm{~B} 3$

$\Delta_{\boldsymbol{f}} \mathbf{H 2 9 8}=\mathbf{6 7 6 . 9} \pm \mathbf{8} \mathbf{~ k J ~ m o l}^{-\mathbf{1}} \mathrm{REF}=\mathrm{Burcat} \mathrm{G} 3 \mathrm{~B} 3$ (thermal electron)

$\left\{\Delta_{f} H 298=663 . \mathrm{kJ} \mathrm{mol}^{-1} \mathrm{REF}=\right.$ Lias et al. ${ }^{10,19}$ (thermal electron) $\}$

5). CAS \# 38607-31-7

$\mathrm{C}_{2} \mathrm{H}_{5} \mathrm{O}+\left[\mathrm{CH}_{2}-\mathrm{CH}_{2}\right] \mathrm{OH}+\quad$ Oxyrene (EthyleneOxide) Protonate (Oxonium) $\sigma=2$ STATWT $=1$

$\mathrm{I}_{\mathrm{A}}=3.9183 \quad \mathrm{I}_{\mathrm{B}}=4.1357 \quad \mathrm{I}_{\mathrm{C}}=6.6672 \mathrm{~g} \mathrm{~cm}^{2} \times 10^{-39}$

$v=610,775,818,887,1014,1104,1158,1220,1233,1236,1307,1507,1539,3185,3188,3293$,

$3303,3599 \mathrm{~cm}^{-1} \mathrm{REF}=\mathrm{B} 3 \mathrm{LYP} / 6-31 \mathrm{G}(\mathrm{d})$

$\Delta_{\boldsymbol{f}} \mathbf{H 2 9 8}=\mathbf{7 1 5 . 0 7} \pm \mathbf{0 . 6 2} \mathbf{~ k J ~ m o l}^{-1} \quad \mathrm{REF}=$ Ruscic ATcT D $2013^{16}$ (thermal electron)

$\left\{\Delta_{f} H 298=716.8 \pm 8 . \mathrm{kJ} \mathrm{mol}^{-1}\right.$ (thermal electron) $\mathrm{REF}=$ Burcat G3B3 $\}$

$\left\{\Delta_{f} H 298=697 . \mathrm{kJ} \mathrm{mol}^{-1} \mathrm{REF}=\right.$ Lias et al. ${ }^{10,19}$ (thermal electron) $\}$

6). CAS \# N/A

$\mathrm{C}_{2} \mathrm{H}_{6} \mathrm{O}^{*}+\mathrm{CH}_{3}-\mathrm{OH}-\mathrm{CH}_{2}{ }^{*}+$ Oxonium, Methyl-Methylene-Protonated radical cation

$\sigma=1 \quad$ STATWT $=2$

$\mathrm{I}_{\mathrm{A}}=1.5384 \quad \mathrm{I}_{\mathrm{B}}=8.2382 \quad \mathrm{I}_{\mathrm{C}}=9.2349 \mathrm{~g} \mathrm{~cm}^{2} \times 10^{-39}$

$v=158,206,350,618,690,792,992,1086,1165,1263,1431,1466,1483,1496,1503,3119,3200$, $3249,3366,3625 \mathrm{~cm}^{-1}$ REF=Burcat G3B3

$\Delta_{\boldsymbol{f}} \mathbf{H 2 9 8}=\mathbf{7 9 7 . 6 3 0} \pm \mathbf{8} . \mathbf{k J ~ m o l}^{-1} \mathrm{REF}=$ Burcat G3B3 (thermal electron) 
7). CAS \# 861994-86-7

$\mathrm{C}_{2} \mathrm{H}_{7} \mathrm{O}+$ Oxonium Dimethyl Ether Protonated cation $\left(\mathrm{CH}_{3}\right)_{2} \mathrm{OH}+\sigma=2$ STATWT $=1$

$\mathrm{I}_{\mathrm{A}}=2.4286 \mathrm{I}_{\mathrm{B}}=9.6793 \mathrm{I}_{\mathrm{C}}=10.8782 \mathrm{~g} \mathrm{~cm}^{2} \times 10^{-39}$

$v=160,208,342,666,806,915,1095,1147,1201,1270,1443,1470,1495(2), 1502,1506,1514$, 3110.6(2),3226.4(2),3242(2),3644 $\mathrm{cm}^{-1} \mathrm{REF}=$ Burcat G3B3

$\Delta_{f} \mathbf{H 2 9 8}=\mathbf{5 6 2 . 2 3 8} \pm \mathbf{8} . \mathbf{~ k J ~ m o l}^{-1} \quad \mathrm{REF}=$ Burcat G3B3 (thermal electron)

$\left\{\Delta_{f} H 298=548 . \mathrm{kJ} \mathrm{mol}^{-1}\right.$ (thermal electron) $\mathrm{REF}=$ Lias et al. $\left.{ }^{10,19}\right\}$

8). CAS \# 763023-19-4

$\mathrm{C}_{2} \mathrm{H}_{7} \mathrm{O}+$ Ethyl Oxonium (Ethanol protonated) $\mathrm{C}_{2} \mathrm{H}_{5}-\mathrm{OH}_{2}+\sigma=1 \quad$ STATWT $=1$

$\mathrm{I}_{\mathrm{A}}=2.7307 \quad \mathrm{I}_{\mathrm{B}}=9.9890 \quad \mathrm{I}_{\mathrm{C}}=11.4822 \quad \operatorname{Ir}\left(\mathrm{CH}_{3}\right)=0.5218 \mathrm{~g} \mathrm{~cm}^{2} \times 10^{-39}$

$\operatorname{ROSYM}=3 \mathrm{~V}(3)=580 . \mathrm{cm}^{-1}$

$v=258,367,654,731,828,930,963,1149,1211,1294,1408,1440,1500,1518,1522,1693,3066$, $3129,3138,3164,3219,3588,3679 \mathrm{~cm}^{-1}$ REF=Burcat G3B3

$\Delta_{\boldsymbol{f}} \mathbf{H 2 9 8}=\mathbf{5 2 7 . 7 6 2} \pm \mathbf{8} . \mathbf{~ k J ~ m o l}^{-1} \quad \mathrm{REF}=$ Burcat G3B3 (thermal electron)

$\left\{\Delta_{f} H 298=513 . \mathrm{kJ} \mathrm{mol}^{-1}\right.$ (thermal electron) $\mathrm{REF}=$ Lias et al. $\left.{ }^{10,19}\right\}$

9). CAS \# N/A

$\mathrm{C}_{3} \mathrm{H}_{7} \mathrm{O}+\mathrm{CH}_{3}\left[\mathrm{CH}-\mathrm{CH}_{2}\right] \mathrm{OH}+$, MethylOxiran Oxonium (PropyleneOxide Protonated)

$\sigma=1 \quad$ STATWT $=1$

$\mathrm{I}_{\mathrm{A}}=5.3157 \quad \mathrm{I}_{\mathrm{B}}=13.3318 \quad \mathrm{I}_{\mathrm{C}}=15.2736 \mathrm{~g} \mathrm{~cm}^{2} \times 10^{-39}$

$v=227,327,383,510,771,865,890,947,1005,1066,1153,1212,1231.5,1240,1295,1421,1446$, $1491.5,1516,1534,3057,3124,3157,3177,3223,3285,3607 \mathrm{~cm}^{-1}$ REF=Burcat G3B3

$\Delta_{\boldsymbol{f}} \mathbf{H 2 9 8}=\mathbf{6 4 8 . 0 0} \pm \mathbf{8} . \mathbf{k J ~ m o l}^{-1} \mathrm{REF}=$ Burcat G3B3 (thermal electron)

$\left\{\Delta_{f} H 298=626 . \mathrm{kJ} \mathrm{mol}^{-1}\right.$ (thermal electron) $\mathrm{REF}=$ Lias et al. $\left.{ }^{10,19}\right\}$ 
10). CAS \# N/A

$\mathrm{C}_{3} \mathrm{H}_{7} \mathrm{O}+\left[\mathrm{CH}_{2}-\mathrm{CH}_{2}-\mathrm{CH}_{2}\right] \mathrm{OH}+$ Cyclo Trimetyleneoxide Protonated $\sigma=2$ STATWT $=1$ $\mathrm{I}_{\mathrm{A}}=7.4619 \mathrm{I}_{\mathrm{B}}=7.8696 \mathrm{I}_{\mathrm{C}}=13.4686 \mathrm{~g} \mathrm{~cm}^{2} \times 10^{-39}$ $v=150,626,722,766,847,850,868,982,1014,1107,1175,1212,1220,1296,1318,1336,1389$, $1516,1521,1548,3137,3143,3146,3192,3231,3234,3628 \mathrm{~cm}^{-1}$ REF=Burcat G3B3

$\Delta_{\boldsymbol{f}} \mathbf{H 2 9 8}=\mathbf{6 4 0 . 8 5} \pm \mathbf{8} . \mathbf{k J ~ m o l}^{-1} \quad \mathrm{REF}=\mathrm{Burcat} \mathrm{G} 3 \mathrm{~B} 3$ (thermal electron)

$\left\{\Delta_{f} H 298=631 . \mathrm{kJ} \mathrm{mol}^{-1} \quad \mathrm{REF}=\right.$ Lias et al. ${ }^{10,19}$ (thermal electron) $\}$

11). CAS \# 130000-05-4

$\mathrm{C}_{3} \mathrm{H}_{8} \mathrm{O}^{*}+$ Oxonium Dimethyl Methylene $\left(\mathrm{CH}_{3}\right)_{2}-\mathrm{O}-\mathrm{CH}_{2}+\sigma=2$ STATWT $=2$ $\mathrm{I}_{\mathrm{A}}=9.0930 \mathrm{I}_{\mathrm{B}}=9.9009 \mathrm{I}_{\mathrm{C}}=17.5349 \mathrm{~g} \mathrm{~cm}^{2} \times 10^{-39}$ $v=135,171,189,271,401,419,671,732,891,1012,1112,1147,1193,1268,1284,1448,1466$, 1493.4(2), 1502,1510,1520,3113(2),3203,3229(2),3242.3(2),3363 $\mathrm{cm}^{-1}$ REF=Burcat G3B3 $\Delta_{\boldsymbol{f}} \mathbf{H 2 9 8}=\mathbf{7 7 2 . 8 2 3} \pm \mathbf{8} \mathbf{~ k J ~ m o l}^{-1}$ REF=Burcat G3B3 (thermal electron)

12). CAS \# 43625-65-6

$\mathrm{C}_{3} \mathrm{H}_{9} \mathrm{O}+$ Trimethyl Oxonium cation $\left(\mathrm{CH}_{3}\right)_{3} \mathrm{O}+\sigma=3$ STATWT $=1$

$\mathrm{I}_{\mathrm{A}}=9.9881 \mathrm{I}_{\mathrm{B}}=9.9933 \mathrm{I}_{\mathrm{C}}=17.8419 \mathrm{~g} \mathrm{~cm}^{2} \times 10^{-39}$ $v=148,195.5,198,299,404(2), 734,930.6(2), 1122,1152.2(2), 1229,1277(2), 1469.7(2), 1487$, 1496.5(2),1515(2),1521.2(2),3101(2),3105,3212,3213(2),3232,3237(2) $\mathrm{cm}^{-1} \mathrm{REF}=\mathrm{G} 3 \mathrm{~B} 3$

$\Delta_{\boldsymbol{f}} \mathbf{H 2 9 8}=\mathbf{5 4 1 . 7 1 6} \pm \mathbf{8} . \mathbf{~ k J ~ m o l}^{-1} \quad \mathrm{REF}=$ Burcat G3B3 (thermal Electron) $\left\{\Delta_{f} H 298=542 . \pm 15 . \mathrm{kJ} \mathrm{mol}^{-1} \mathrm{REF}=\text { Neguyen Bouchoux (thermal electron) }\right\}^{1}$ 
13). CAS \# N/A

$n-\mathrm{C}_{3} \mathrm{H}_{9} \mathrm{O}+$ n-Protonated Propanol cation (Oxonium) $\mathrm{C}_{3} \mathrm{H}_{7} \mathrm{OH}_{2}+\sigma=1$ STATWT $=1$

$\mathrm{I}_{\mathrm{A}}=3.4407 \quad \mathrm{I}_{\mathrm{B}}=23.8688 \quad \mathrm{I}_{\mathrm{C}}=25.5576 \quad \mathrm{I}_{\mathrm{r}}\left(\mathrm{CH}_{3}\right)=0.5214 \mathrm{~g} \mathrm{~cm}^{2} \times 10^{-39}$

ROSYM $=3 \quad V_{(3)}=3200 \mathrm{~cm}^{-1} \quad \mathrm{I}_{\mathrm{r}}\left(\mathrm{H}_{2} \mathrm{OCH}_{2-}\right)=4.718 \mathrm{~g} \mathrm{~cm}^{2} \times 10^{-39}$

ROSYM $=1 \quad V_{(3)}=2000 \mathrm{~cm}^{-1}$

$v=234,249,401,687,732,786,885,914,984,1017,1161,1203,1273,1336,1349,1410,1450$,

1519(2),1528,1538,1690,3053,3073,3113,3130,3147,3160,3213,3589,3681 $\mathrm{cm}^{-1}$ REF=G3B3

$\Delta_{f} \mathbf{H 2 9 8}=\mathbf{5 0 1 . 8 5} \pm \mathbf{8} . \mathbf{~ k J ~ m o l}^{-1} \quad \mathrm{REF}=\mathrm{Burcat} \mathrm{G} 3 \mathrm{~B} 3$ (thermal Electron)

$\left\{\Delta_{f} H 298=482 . \mathrm{kJ} \mathrm{mol}^{-1} \quad \mathrm{REF}=\right.$ Lias et al. ${ }^{10,19}$ (thermal electron) $\}$

14). CAS \# N/A

$i-\mathrm{C}_{3} \mathrm{H}_{9} \mathrm{O}+$ i-Protonated Propanol cation (Oxonium) $\left(\mathrm{CH}_{3}\right)_{2} \mathrm{CHOH}_{2}+\sigma=2$ STATWT $=1$

$\mathrm{I}_{\mathrm{A}}=10.9457 \mathrm{I}_{\mathrm{B}}=11.1225 \mathrm{I}_{\mathrm{C}}=19.1610 \mathrm{I}_{\mathrm{r}}\left(\mathrm{H}_{2} \mathrm{OCH}-\right)=3.947 \mathrm{~g} \mathrm{~cm}^{2} \times 10^{-39}$

ROSYM=1 $V_{(3)}=2000 . \mathrm{cm}-1$

$v=228,265,350,354,428,544,741,869,908,936,962,1108,1187,1230,1343.6,1439,1451$,

$1495,1516(3), 1690,3060,3065,3128,3132,3141,3158,3169,3593,3689 \mathrm{~cm}^{-1} \mathrm{REF}=\mathrm{G} 3 \mathrm{~B} 3$

$\Delta_{\boldsymbol{f}} \mathbf{H 2 9 8}=\mathbf{4 8 1 . 8} \pm \mathbf{8} . \mathbf{~ k J ~ m o l}^{-1} \quad \mathrm{REF}=$ Burcat G3B3 (thermal Electron)

$\left\{\Delta_{f} H 298=463 . \mathrm{kJ} \mathrm{mol}^{-1}\right.$ REF=Lias et al. ${ }^{10,19}$ (thermal electron) $\}$ 
15). CAS \# N/A

$\mathrm{C}_{4} \mathrm{H}_{9} \mathrm{O}+$ TetrahydroFuran-Oxonium $\left[\mathrm{CH}_{2}-\mathrm{CH}_{2}-\mathrm{CH}_{2}-\mathrm{CH}_{2}\right] \mathrm{OH}+\sigma=2$ STATWT $=1$

$\mathrm{I}_{\mathrm{A}}=12.6482 \quad \mathrm{I}_{\mathrm{B}}=12.8351 \quad \mathrm{I}_{\mathrm{C}}=22.4090 \mathrm{~g} \mathrm{~cm}^{2} \times 10^{-39}$

$v=125,262,521,559,683,738,781,845,901,917,953,989,1058,1138,1194,1209,1243,1274$,

$1333,1346,1384,1398,1413,1512,1517,1526,1543,3096,3102,3127,3140,3150,3158,3213$, $3216,3676 \mathrm{~cm}^{-1} \mathrm{REF}=$ Burcat G3B3

$\Delta_{\boldsymbol{f}} \mathbf{H 2 9 8}=\mathbf{5 2 9 . 0 7} \pm \mathbf{8} \mathbf{~ k J ~ m o l}^{-1}$ REF=Burcat G3B3 (thermal electron)

$\left\{\Delta_{f} H 298=520 . \mathrm{kJ} \mathrm{mol}^{-1} \quad \mathrm{REF}=\right.$ Lias et al. ${ }^{10,19}$ (thermal electron) $\}$

16). CAS \# 44209-06-5

$\mathrm{C}_{4} \mathrm{H}_{11} \mathrm{O}+$ Oxonium-Ethyl-Dimethyl $\left(\mathrm{CH}_{3}\right)_{2}-\mathrm{O}-\mathrm{C}_{2} \mathrm{H}_{5}+\sigma=2$ STATWT $=1$

$\mathrm{I}_{\mathrm{A}}=10.9742 \mathrm{I}_{\mathrm{B}}=23.9455 \mathrm{I}_{\mathrm{C}}=31.2570 \mathrm{~g} \mathrm{~cm}^{2} \times 10^{-39}$

$\operatorname{Ir}\left(\mathrm{CH}_{3}\right)=0.52354 \mathrm{~g} \mathrm{~cm}^{2} \times 10^{-39} \mathrm{ROSYM}=3 \quad \mathrm{~V}(3)=411 \mathrm{~cm}-1$

$v=163,185,228,288,334,403,440,687,820,857,955,984,1115,1143,1151,1205,1231,1269$,

$1329,1416,1445,1469,1496(2), 1501,1512,1516,1522(2), 1533,3075,3100,3104,3110,3142$,

$3163,3193,3210,3212,3232,3237 \mathrm{~cm}^{-1} \mathrm{REF}=$ Burcat G3B3

$\Delta_{f} \mathbf{H 2 9 8}=\mathbf{4 9 7 . 9 8} \pm \mathbf{8} \mathbf{~ k J ~ m o l}^{-1} \mathrm{REF}=\mathrm{Burcat} \mathrm{G} 3 \mathrm{~B} 3$ (thermal electron)

$\left\{\Delta_{f} H 298=496.2 \pm 15 \mathrm{~kJ}\right.$ REF $=$ Tu Holmes ${ }^{2}$ (thermal electron) $\}$ 
17). CAS \# 1476849-22-5

$\mathrm{C}_{4} \mathrm{H}_{11} \mathrm{O}+$ Protonated DiEthyl Ether Oxonium $\left(\mathrm{C}_{2} \mathrm{H}_{5}\right)_{2} \mathrm{OH}+\sigma=2$ STATWT $=1$

$\mathrm{I}_{\mathrm{A}}=8.1467 \mathrm{I}_{\mathrm{B}}=34.3188 \mathrm{I}_{\mathrm{C}}=34.6674 \mathrm{~g} \mathrm{~cm}^{2} \times 10^{-39}$

$\left[\operatorname{Ir}\left(\mathrm{CH}_{3}\right)=0.52354 \mathrm{~g} \mathrm{~cm}^{2} \times 10^{-39} \mathrm{ROSYM}=3 \mathrm{~V}(3)=680 . \mathrm{cm}-1\right] \mathrm{x} 2$

$v=214,259,283,401,404,630,716,775,811,833,973,980,1090,1126,1150,1216,1296,1323$, $1386,1400,1442,1447,1456,1507,1512.2(2), 1522,1527(2), 3065,3074,3123,3132,3142.5(2)$,

$3151,3164,3195,3213,3651 \mathrm{~cm}^{-1}$ REF=Burcat G3B3

$\Delta_{\boldsymbol{f}} \mathbf{H 2 9 8}=\mathbf{4 6 8 . 0 7 7} \pm \mathbf{8} . \mathbf{~ k J ~ m o l}^{-1}$ REF=Burcat G3B3 (thermal Electron)

$\left\{\Delta_{f} H 298=446 \mathrm{~kJ} \mathrm{~mol}^{-1}\right.$ (thermal electron) $\mathrm{REF}=$ Lias et al. $\left.{ }^{10,19}\right\}$

18). CAS \# N/A

$\mathrm{C}_{5} \mathrm{H}_{11} \mathrm{O}+\left[\mathrm{CH}_{2}-\mathrm{CH}_{2}-\mathrm{CH}_{2}-\mathrm{CH}_{2}-\mathrm{CH}_{2}\right] \mathrm{OH}+$ PentaHydroxyPyran Protonated (CycloPentaMethyleneOxide Oxonium) $\sigma=2$ STATWT $=1$

$\mathrm{I}_{\mathrm{A}}=18.9879 \mathrm{I}_{\mathrm{B}}=20.206 \mathrm{I}_{\mathrm{C}}=33.1435 \mathrm{~g} \mathrm{~cm}^{2} \times 10^{-39}$

$v=87,217,273,411,474,584,644,737,768,813,841,885,923,969,999,1028,1094,1172,1175$, $1219,1275,1305,1318,1363,1383,1400,1411,1418,1439,1512,1516,1523,1531,1540,3058$, 3078.2(2),3118(2),3134,3140,3143,3205.5(2),3665 $\mathrm{cm}^{-1}$ REF=Burcat G3B3

$\Delta_{f} \mathbf{H 2 9 8}=\mathbf{5 1 0 . 9 6 7} \pm \mathbf{8} . \mathbf{k J ~ m o l}^{-1} \mathrm{REF}=$ Burcat G3B3 (thermal Electron)

$\left\{\Delta_{f} H 298=578 \mathrm{~kJ} \mathrm{~mol}^{-1}\right.$ (thermal electron) $\mathrm{REF}=$ Lias et al. $\left.{ }^{10,19}\right\}$ 
19). CAS \# 44387-70-4

$\mathrm{C}_{5} \mathrm{H}_{13} \mathrm{O}+$ Oxonium DiethylMethyl $\left(\mathrm{C}_{2} \mathrm{H}_{5}\right)_{2} \mathrm{OCH}_{3} \sigma=2$ STATWT $=1$

$\mathrm{I}_{\mathrm{A}}=20.8679 \mathrm{I}_{\mathrm{B}}=30.65559 \mathrm{I}_{\mathrm{C}}=42.9658 \mathrm{~g} \mathrm{~cm}^{2} \times 10^{-39}$

$\left[\operatorname{Ir}\left(\mathrm{CH}_{3}\right)=0.52354 \mathrm{~g} \mathrm{~cm}^{2} \times 10^{-39} \mathrm{ROSYM}=3 \mathrm{~V}(3)=1254 . \mathrm{cm}^{-1}\right] \mathrm{x} 2$

$\operatorname{Ir}\left(\mathrm{CH}_{3}\right)=0.52354 \mathrm{~g} \mathrm{~cm}^{2} \times 10^{-39} \mathrm{ROSYM}=3 \mathrm{~V}(3)=380 . \mathrm{cm}^{-1}$

$v=209,245,273,302,391,437,458,654,791,815,826,937,982,990,1113,1116,1151,1186,1219$, $1238,1319,1343,1403,1418,1446(2), 1490,1503,1506,1510,1521(2), 1525,1533,1540$,

3073.4(2),3105,3117,3135,3142,3147,3154,3160,3188,3211(2),3226 $\mathrm{cm}^{-1}$ REF=Burcat G3B3

$\Delta \boldsymbol{f H 2 9 8}=\mathbf{4 5 5 . 7 2 2} \pm \mathbf{8} . \mathbf{~ k J ~ m o l}^{-1}$ (thermal electron) $\mathrm{REF}=$ Burcat G3B3

20). CAS \# 24935-24-8

$\mathrm{C}_{6} \mathrm{H}_{15} \mathrm{O}+$ Oxonium TriEthyl $\left(\mathrm{C}_{2} \mathrm{H}_{5}\right)_{3} \mathrm{O}+\sigma=3$ STATWT $=1$

$\mathrm{I}_{\mathrm{A}}=27.8116 \mathrm{I}_{\mathrm{B}}=43.9193 \mathrm{I}_{\mathrm{C}}=62.5374 \quad\left[\mathrm{Ir}\left(\mathrm{CH}_{3}\right)=0.52056 \mathrm{~g} \mathrm{~cm}^{2} \times 10^{-39}\right.$

ROSYM=3 V(3)=780. cm-1]x3

$v=178,216,252,281,323(2), 369,418,468,782,808,811,856,861,977,980,1010,1109,1121,1149$, $1157,1210,1221,1300,1337(2), 1386,1413,1441,1446,1450,1463,1507.4(2), 1514(2), 1521$,

1527.5(2),1537,1541,3075.3(2),3077,3102,3107,3127,3143.5(2),3149,3160,3163(2),3183,

3193(2) $\mathrm{cm}^{-1}$ REF=Burcat G3B3

$\Delta_{\boldsymbol{f}} \mathbf{H 2 9 8}=\mathbf{4 1 4 . 6 9 8} \pm \mathbf{8} \mathbf{~ k J ~ m o l}^{-1} \mathrm{REF}=$ Burcat G3B3 thermal electron

$\left\{\Delta_{f} H 298=412.2 \mathrm{~kJ}\right.$ REF $=\mathrm{Tu} \&$ Holmes $^{2}$ thermal electron $\}$.

* Denotes a radical cation Oxonium with STATWT $=2$ 
Table 2 Thermochemical data of oxonium cations at 298.15 and $0 \mathrm{~K}$.

\begin{tabular}{|c|c|c|c|c|c|c|c|}
\hline Oxonium Compound & $\begin{array}{l}\text { Mol. } \\
\text { Wgt. }\end{array}$ & $\begin{array}{l}\Delta_{\mathrm{f}} \mathrm{H}_{298} \\
\mathrm{~kJ} / \mathrm{mol}\end{array}$ & $\begin{array}{c}\Delta_{\mathrm{f}} \mathrm{H}_{0} \\
\mathrm{~kJ} / \mathrm{mol}\end{array}$ & $\begin{array}{c} \pm \\
\mathrm{kJ} / \mathrm{mol}\end{array}$ & \begin{tabular}{|c|}
$C_{p 298}$ \\
$\mathrm{~J} / \mathrm{mol} /$ \\
$\mathrm{K}$
\end{tabular} & $\begin{array}{c}\mathrm{S}_{298} \\
\mathrm{~J} / \mathrm{mol} / \mathrm{K}\end{array}$ & $\begin{array}{l}\mathrm{H}_{298}-\mathrm{H}_{0} \\
\mathrm{~kJ} / \mathbf{m o l}\end{array}$ \\
\hline $\mathrm{CHOH}_{2}+$ Methylidene oxonium & 31.03337 & 1042.180 & 1062.60 & \pm 0.7 & 43.951 & 231.894 & 10.760 \\
\hline $\mathrm{CH}_{2} \mathrm{OH}_{2}{ }^{*}+$ Methylene oxonium & 32.04131 & 825.900 & 830.046 & \pm 4.5 & 52.353 & 247.500 & 11.987 \\
\hline $\mathrm{CH}_{3}-\mathrm{OH}_{2}+$ Rydberg Cation & 33.04925 & 583.706 & 585.970 & \pm 8 & 51.102 & 244.423 & 11.902 \\
\hline $\begin{array}{l}\mathrm{CH}_{3}-\mathrm{O}=\mathrm{CH}_{2}+\text { Methyl Methylene } \\
\text { oxoniu }\end{array}$ & 45.05995 & 676.909 & 678.982 & \pm 8 . & 59.088 & 264.746 & 13.149 \\
\hline $\begin{array}{l}{\left[\mathrm{CH}_{2}-\mathrm{CH}_{2}\right] \mathrm{OH}+\text { Protonated }} \\
\text { Oxirane }\end{array}$ & 45.05995 & $715.07^{\mathrm{d}}$ & 725.040 & \pm 0.62 & 52.329 & 246.649 & 11.266 \\
\hline $\begin{array}{l}\mathrm{CH}_{3} \mathrm{OH}-\mathrm{CH}_{2}{ }^{*}+\mathrm{Me} \text { Methylene } \\
\text { Proton }\end{array}$ & 46.06789 & 797.630 & 801.883 & \pm 8 . & 72.130 & 282.717 & 15.204 \\
\hline $\begin{array}{l}\left(\mathrm{CH}_{3}\right)_{2} \mathrm{OH}+\text { Dimethyl Proton } \\
\text { oxonium }\end{array}$ & 47.07583 & 562.238 & 570.996 & \pm 8 . & 69.946 & 273.423 & 14.933 \\
\hline $\mathrm{C}_{2} \mathrm{H}_{5}-\mathrm{OH}_{2}+$ Diethyl oxonium & 47.07583 & 527.762 & 536.286 & \pm 8 & 72.680 & 280.690 & 15.171 \\
\hline $\mathrm{C} 3 \mathrm{H} 7 \mathrm{O}+\mathrm{Me}$ Oxirane protonated & 59.08653 & 648.00 & 663.775 & \pm 8 & 76.735 & 284.409 & 14.821 \\
\hline $\begin{array}{l}\mathrm{C} 3 \mathrm{H} 7 \mathrm{O}+\mathrm{CyTrimethyleneoxide} \\
\text { Protonated }\end{array}$ & 59.08653 & 640.85 & 657.96 & \pm 8 . & 68.609 & 271.860 & 13.527 \\
\hline $\begin{array}{l}\left(\mathrm{CH}_{3}\right)_{2} \mathrm{O}-\mathrm{CH}_{2}{ }^{*}+\text { Dimethyl } \\
\text { Methylene }\end{array}$ & 60.09447 & 772.823 & 783.086 & \pm 8 . & 93.481 & 312.207 & 18.716 \\
\hline $\begin{array}{l}\left(\mathrm{CH}_{3}\right)_{3} \mathrm{O}+\text { Trimethyl oxonium } \\
\text { cation }\end{array}$ & 61.10241 & 541.716 & 556.731 & \pm 8 & 90.997 & 299.987 & 18.197 \\
\hline $\begin{array}{l}n-\mathrm{C}_{3} \mathrm{H}_{7} \mathrm{OH}_{2}+n \text {-Protonated } \\
\text { Propanol }\end{array}$ & 61.10241 & 501.846 & 495.649 & \pm 8 & 92.343 & 316.534 & 17.760 \\
\hline $\begin{array}{l}i-\mathrm{C}_{3} \mathrm{H}_{7} \mathrm{OH}_{2}+i \text {-Protonated } \\
\text { Propanol }\end{array}$ & 61.10241 & 471.809 & 465.612 & \pm 8 & 97.788 & 315.417 & 18.657 \\
\hline
\end{tabular}




\begin{tabular}{|c|c|c|c|c|c|c|c|}
\hline $\begin{array}{l}\left(\mathrm{CH}_{3}\right)_{2} \mathrm{O}-\mathrm{C}_{2} \mathrm{H}_{5}+\text { DiMethyl Ethyl } \\
\text { oxoni }\end{array}$ & 75.12899 & 497.984 & 519.411 & \pm 8 . & 110.480 & 331.076 & 21.307 \\
\hline $\begin{array}{l}\left(\mathrm{C}_{2} \mathrm{H}_{5}\right)_{2} \mathrm{OH}+\text { DiEthyl oxonium } \\
\text { cation }\end{array}$ & 75.12899 & 468.077 & 489.895 & \pm 8 . & 112.598 & 326.792 & 20.917 \\
\hline $\begin{array}{l}\left(\mathrm{CH}_{2} \mathrm{CH}_{2} \mathrm{CH}_{2} \mathrm{CH}_{2}\right) \mathrm{OH}+ \\
\text { cy Tetrahydrofuran Oxonium }\end{array}$ & 73.11311 & 529.067 & 553.199 & \pm 8 . & 86.994 & 293.796 & 15.892 \\
\hline $\begin{array}{l}\mathrm{C} 5 \mathrm{H} 11 \mathrm{O}+\text { cy PentahydroxyPyran } \\
\text { protonate } \\
\left(\mathrm{CH}_{2} \mathrm{CH}_{2} \mathrm{CH}_{2} \mathrm{CH}_{2} \mathrm{CH}_{2}\right) \mathrm{OH}+\end{array}$ & 87.13969 & 510.967 & 541.740 & \pm 8 & 106.110 & 317.820 & 18.644 \\
\hline $\begin{array}{l}\left(\mathrm{C}_{2} \mathrm{H}_{5}\right)_{2} \mathrm{OCH}_{3}+\text { Diethyl Methyl } \\
\text { oxoniu }\end{array}$ & 89.15557 & 455.722 & 484.205 & \pm 8 . & 132.290 & 349.675 & 23.773 \\
\hline $\begin{array}{l}\left(\mathrm{C}_{2} \mathrm{H}_{5}\right)_{3} \mathrm{O}+\text { TriEthyl oxonium } \\
\text { cation }\end{array}$ & $\begin{array}{r}103.1821 \\
5\end{array}$ & 414.698 & 449.283 & \pm 8 . & 155.400 & 370.702 & 27.192 \\
\hline
\end{tabular}

* Oxoniums that are radical cations or distonic ions.

\section{DISCUSSION}

The Ideal Gas Thermochemistry of 20 carbon oxonium cations have been calculated and set in polynomial form for the first time. Four of the enthalpies of formation calculated, are not mentioned in the literature. Nine of them have no CAS numbers. Most of the compounds are protonated specie but three of them (\#2,6, and 11) are open shell radical cations/distonic ions, with statistical weight of 2 , (doubletes, instead of singlets for the rest).

The enthalpies of formation of the second oxonium and of the fifth oxonium - protonated oxirene- were calculated in a rigorous way by Ruscic in the ATcT tables of $2013^{16}$. The ATcT values are considered as the most reliable values, which unlike the traditional sequential approach, derives reliable, and internally consistent thermochemical values by simultaneously analyzing and solving the underlying Thermo-chemical Network (TN). The TN is constructed from the available thermochemical interdependencies relevant to the targeted species, such as measured ionization energies, electron affinities, reaction enthalpies, bond dissociation energies, constants of equilibria etc. The ATcT allows mixing experimental and computational results. Thus the data of each species is dependent not only on its own experimental data, but also on the experimental values of other specie currently in the table. Therefor these values got priority when choosing the 
enthalpy of formation of the specie. For all the rest we preferred our DFT G3B3 calculations for reasons described in the calculation paragraph. It should be noted that our G3B3 estimates are less than $\pm 2 \mathrm{~kJ} \mathrm{~mol}^{-1}$ (less than $0.5 \mathrm{kcal} \mathrm{mol}^{-1}$ ) apart from the ATcT values and also of the other literature values we have found, except those of Brown and Eraslan $^{17}$ and of Lias et al. ${ }^{10,19}$. This gives us the assurance that our G3B3 values are acceptable with actual errors bars below the standard high error bar of the protocol i.e. $\pm 8 . \mathrm{kJ} \mathrm{mol}^{-1}$. The 13 old estimates of Lias et al. ${ }^{10,19}$ were 9 to $30 \mathrm{~kJ} \mathrm{~mol}^{-1}$ lower than the G3B3 and other references. In 1984 Lias Liebman \& Levine ${ }^{19}$ published the GIANT table of heats of formation of ions followed by the Lias et $a l^{10}$ that repeated most of the earlier data. Their data were estimated when $a b$ initio calculations were not well established, and were calculated from the enthalpy of formation of the corresponding non protonated specie or by the proton affinity method. Brown and Eraslan ${ }^{17}$ suggest for the third species (the Rydberg cation) an estimate only, which is at the edge of our G3B3 error bar .

\section{Individual Oxonium Compounds}

1. $\mathrm{CHOH}_{2}+$ Oxonium Methylidene protonated cation This is one of the simplest carbon oxonium cations. The thermochemistry of this compound was never calculated, and it has no CAS number. Our G3B3 calculation is $\Delta_{f} H 298=1042.05 \pm 8 . \mathrm{kJ} \mathrm{mol}^{-1}$ (thermal electron). The G3B3 calculation was repeated three times with different atom positions and all gave the same results $\left( \pm 0.03 \mathrm{~kJ} \mathrm{~mol}^{-1}\right)$

2. $\mathrm{CH}_{2} \mathrm{OH}_{2}{ }^{*}+$ Methylene Oxonium Radical cation This radical cation is a distonic ion with an open shell species and a doublet ground state term. The enthalpy of formation of this oxonium is displayed in Ruscic's ATcT tables ${ }^{16}$ from 2013 as $\Delta_{f} H 298=825.9 \pm 4.1 \mathrm{~kJ}$ $\mathrm{mol}^{-1}$. It has no CAS number. The number quoted by Lias et al ${ }^{10,19}$ is erroneous. Our G3B3 calculation gives $\Delta_{f} H 298=826.75 \pm 8 . \mathrm{kJ} \mathrm{mol}^{-1}$ in excellent agreement with the ATcT calculation. The Lias et al ${ }^{10,19}$ calculation reads $\Delta_{f} H 298=815 . \pm 8 . \mathrm{kJ} \mathrm{mol}^{-1} 11 \mathrm{~kJ}$ $\mathrm{mol}^{-1}$, lower (all thermal electron).

3. $\mathrm{CH}_{3} \mathrm{OH}_{2}+$ Protonated Methanol cation (Methyl Oxonium) is also known as Rydberg cation. The enthalpy of formation was calculated with the G3B3 method as $\Delta_{f} H 298=583.706 \pm 8 . \mathrm{kJ} \mathrm{mol}^{-1}$. There exists an estimate from 1988 by Brown and Eraslan ${ }^{17}$ as $\Delta_{f} H 298=592 . \mathrm{kJ} \mathrm{mol}^{-1}$ and Lias et al ${ }^{10,19}$ estimate $\Delta_{f} H 298=573 . \mathrm{kJ} \mathrm{mol}^{-1} 11 \mathrm{~kJ} \mathrm{~mol}^{-1}$ lower than the G3B3 calculation (all thermal electron convention). 
4. $\mathrm{CH}_{3}-\mathrm{O}=\mathrm{CH}_{2}+$ Methyl Methylene Oxonium This specie is reported as oxonium although the oxygen atom is connected to only two carbon atoms. It is assumed that the methylene carbon is connected with a double bond. Our G3B3 calculation gives $\Delta_{f} H 298=676.909 \pm 8 . \mathrm{kJ} \mathrm{mol}^{-1}$. The Lias et al ${ }^{10,19}$ estimate is $\Delta_{f} H 298=663 . \mathrm{kJ} \mathrm{mol}^{-1} 14 \mathrm{~kJ}$ $\mathrm{mol}^{-1}$ lower (both thermal electron).

5. $\left[\mathrm{CH}_{2}-\mathrm{CH}_{2}\right] \mathrm{OH}+$ Oxyrene (EthyleneOxide) Protonated (Oxonium) This is a cyclo protonated compound in our oxonium list. The enthalpy of formation of this oxonium is displayed in Ruscic's ATcT tables ${ }^{16}$ from 2013 as $\Delta_{f} H 298=715.07 \pm 0.62 \mathrm{~kJ} \mathrm{~mol}^{-1}$. Our G3B3 calculation is in excellent agreement $\Delta_{f} H 298=716.8 \pm 8 . \mathrm{kJ} \mathrm{mol}^{-1}$ and the Lias et $a l^{10,19}$ estimate is $18 \mathrm{~kJ} \mathrm{~mol}^{-1}$ lower $\Delta_{f} H 298=697 . \mathrm{kJ} \mathrm{mol}^{-1}$ (all thermal electron).

6. $\mathrm{CH}_{3}-\mathrm{OH}-\mathrm{CH}_{2}{ }^{*}+$ Methyl Methylene Protonated radical Cation Oxonium The thermochemistry of this cation was not reported in the literature, and no CAS number was assigned to it. This is an open shell distonic ion (radical cation) with a doublet ground state term. The enthalpy of formation calculated by the G3B3 method is $\Delta_{f} H 298=797.63 \pm 8 . \mathrm{kJ} \mathrm{mol}^{-1}$ (thermal electron).

7. $\left(\mathrm{CH}_{3}\right)_{2}-\mathrm{OH}+$ Oxonium, DiMethylEther Protonated Cation The enthalpy of formation calculated by the G3B3 method is $\Delta_{f} H 298=562.24 \pm 8 . \mathrm{kJ} \mathrm{mol}^{-1}$ (thermal electron). The old value estimate of Lias et al ${ }^{10,19}$ gives $\Delta_{f} H 298=548 . \mathrm{kJ} \mathrm{mol}^{-1} 14 \mathrm{~kJ} \mathrm{~mol}^{-1}$ lower.

8. $\mathrm{C}_{2} \mathrm{H}_{5} \mathrm{OH}_{2}+$ Protonated Ethanol Cation Oxonium The enthalpy of formation calculated by the G3B3 method is $\Delta_{f} H 298=527.76 \pm 8 . \mathrm{kJ} \mathrm{mol}^{-1}$ (thermal electron). The Lias et al ${ }^{10,19}$ old value gives $\Delta_{f} H 298=513 . \mathrm{kJ} \mathrm{mol}^{-1} 15 \mathrm{~kJ} \mathrm{~mol}^{-1}$ lower.

9. $\mathrm{CH}_{3}\left[\mathrm{CH}-\mathrm{CH}_{2}\right] \mathrm{OH}+$, Methy lOxiran Oxonium (PropyleneOxide Protonated) The enthalpy of formation calculated by the G3B3 method is $\Delta_{f} H 298=648.00 \pm 8 . \mathrm{kJ} \mathrm{mol}^{-1}$. This oxonium was reported by Lias et al. ${ }^{10,19} \Delta_{f} H 298=626 . \mathrm{kJ} \mathrm{mol}^{-1}, 22 \mathrm{~kJ} \mathrm{~mol}^{-1}$ lower (thermal electron). The compound has no CAS number. 
10. $\left[\mathrm{CH}_{2}-\mathrm{CH}_{2}-\mathrm{CH}_{2}\right] \mathrm{OH}+$ Protonated Oxetane cation, Cyclo Trimetyleneoxide Oxonium The enthalpy of formation calculated by the G3B3 method is $\Delta_{f} H 298=640.85 \pm 8 . \mathrm{kJ} \mathrm{mol}^{-1}$. The compound was estimated by Lias et $a l^{10,19}$ as $\Delta_{f} H 298=631 . \mathrm{kJ} \mathrm{mol}^{-1}, 10 \mathrm{~kJ} \mathrm{~mol}^{-1}$ (thermal electron). It has no CAS number.

11. $\left(\mathrm{CH}_{3}\right)_{2} \mathrm{O}-\mathrm{CH}_{2}{ }^{*}+$ Dimethyl Methylene Oxonium The thermochemistry of this cation was not reported in the literature. It is a radical cation, an open shell distonic ion with a doublet ground state term. The enthalpy of formation calculated by the G3B3 method is $\Delta_{f} H 298=772.823 \pm 8 . \mathrm{kJ} \mathrm{mol}^{-1}$ (thermal electron).

12. $\left(\mathrm{CH}_{3}\right)_{3} \mathrm{O}+$ TriMethyl Oxonium The enthalpy of formation calculated by the G3B3 method is $\Delta_{f} H 298=541.716 \pm 8 . \mathrm{kJ} \mathrm{mol}^{-1}$. This oxonium was reported by Neguyen and Bouchoux ${ }^{1}$ as $\Delta_{f} H 298=542.2 \pm 15 . \mathrm{kJ} \mathrm{mol}^{-1}$ (both thermal electron). Although they made a rough estimate, it is in excellent agreement with our result.

13. $n-\mathrm{C}_{3} \mathrm{H}_{7} \mathrm{OH}_{2}+$ Protonated n-Propanol Oxonium This compound has no CAS number. Its enthalpy of formation calculated by the G3B3 method is $\Delta_{f} H 298=501.85 \pm 8 . \mathrm{kJ} \mathrm{mol}^{-1}$. The Lias et al ${ }^{10,19}$ estimate was $\Delta_{f} H 298=482 . \mathrm{kJ} \mathrm{mol}^{-1}, 20 \mathrm{~kJ} \mathrm{~mol}^{-1}$ lower. (all thermal electron).

14. $\left(\mathrm{CH}_{3}\right)_{2} \mathrm{CHOH}_{2}+$ Protonated iso-Propanol Oxonium This compound has no CAS number.

Its enthalpy of formation calculated by the G3B3 method is $\Delta_{f} H 298=481.8 \pm 8 . \mathrm{kJ} \mathrm{mol}^{-1}$. The Lias et $\mathrm{al}^{10,19}$ estimate was $\Delta_{f} H 298=463 . \mathrm{kJ} \mathrm{mol}^{-1} 19 \mathrm{~kJ} \mathrm{~mol}^{-1}$ lower. (all thermal electron).

15. $\left[\mathrm{CH}_{2}-\mathrm{CH}_{2}-\mathrm{CH}_{2}-\mathrm{CH}_{2}\right] \mathrm{OH}+$ TetrahydroFuran Protonated Cation (CycloTetramethyleneOxide Oxonium) The enthalpy of formation of this cyclo protonated compound that was calculated by the G3B3 method is $\Delta_{f} H 298=529.067 \pm 8 . \mathrm{kJ} \mathrm{mol}^{-1}$. The old Lias et al ${ }^{10,19}$ estimate was $\Delta_{f} H 298=520 . \mathrm{kJ} \mathrm{mol}^{-1}, 9 \mathrm{~kJ} \mathrm{~mol}^{-1}$ lower. (all thermal electron).

16. $\mathrm{C}_{2} \mathrm{H}_{5}-\mathrm{O}-\left(\mathrm{CH}_{3}\right)_{2}+$ Ethyl DiMethyl Oxonium The enthalpy of formation calculated by the G3B3 method is $\Delta_{f} H 298=497.984 \pm 8 . \mathrm{kJ} \mathrm{mol}^{-1}$. This oxonium was reported by $\mathrm{Tu}$ and Holmes ${ }^{2}$ as $\Delta_{f} H 298=496.2 \mathrm{~kJ} \mathrm{~mol}^{-1}$, in excellent agreement with the G3B3 value (both thermal electron). 
17. $\left(\mathrm{C}_{2} \mathrm{H}_{5}\right)_{2} \mathrm{OH}+$ Protonated DiEthyl Ether Oxonium The enthalpy of formation calculated by the G3B3 method is $\Delta_{f} H 298=468.077 \pm 8 . \mathrm{kJ} \mathrm{mol}^{-1}$ (thermal electron). The Lias et al ${ }^{10,19}$ old estimate gives $\Delta_{f} H 298=446 . \mathrm{kJ} \mathrm{mol}^{-1}, 22 . \mathrm{kJ} \mathrm{mol}^{-1}$ lower.

18. $\left[\mathrm{CH}_{2}-\mathrm{CH}_{2}-\mathrm{CH}_{2}-\mathrm{CH}_{2}-\mathrm{CH}_{2}\right] \mathrm{OH}+$ PentaHydroxyPyran Protonated (CycloPentaMethyleneOxide Oxonium) It has no CAS number. The enthalpy of formation calculated by the G3B3 method is $\Delta_{f} H 298=510.967 \pm 8 . \mathrm{kJ} \mathrm{mol}^{-1}$. The old Lias et $\mathrm{al}^{10,19}$ estimate was $\Delta_{f} H 298=478 . \mathrm{kJ}$ $\mathrm{mol}^{-1}, 33 \mathrm{~kJ} \mathrm{~mol}^{-1}$ lower. (thermal electron).

19. $\mathrm{CH}_{3}-\mathrm{O}-\left(\mathrm{C}_{2} \mathrm{H}_{5}\right)_{2}+$ Methyl DiEthyl Oxonium The thermochemistry of this cation is not reported in the literature. The enthalpy of formation calculated by the G3B3 method is $\Delta_{f} H 298=455.722 \pm 8 . \mathrm{kJ} \mathrm{mol}^{-1}$ (thermal electron).

20. $\left(\mathrm{C}_{2} \mathrm{H}_{5}\right)_{3} \mathrm{O}+$ TriEthyl Oxonium The enthalpy of formation calculated by the G3B3 method is $\Delta_{f} H 298=414.598 \pm 8 . \mathrm{kJ} \mathrm{mol}^{-1}$. This oxonium compound was reported by $\mathrm{Tu}$ and Holmes $^{2}$ as $\Delta_{f} H 298=412.2 \mathrm{~kJ} \mathrm{~mol}^{-1}$ in good agreement with our value. (both thermal electron).

\section{CONCLUSION}

The thermochemical data of 20 Oxonium cation compounds were calculated for the first time with the DFT G3B3 protocol and are provided as 7 term NASA polynomials (in the supplement) for the use of chemical kineticists. The results were compared to existing estimates or calculations available.

Nine term NASA polynomials of the 20 oxonium specie can be found in the author's thermochemical database. ${ }^{20}$

\section{Acknowledgement}

The authors wish to thank the free table sources such as The NIST Chemistry Webbook, the Wikipedia, and ATcT, without which it would have been harder to perform this research. 


\section{Supporting Information}

Table S1 contains the 7-term NASA polynomials of the 20 oxonium cations discussed in this article.

\section{References}

1. Neguyen, M.T.; Bouchoux, G. Theoretical Analysis of the methane Elimination from Oxonium cations $\left[\mathrm{R}_{3} \mathrm{O}\right]+$ J. Phys Chem 1996, 100, 2080-2093.

2. Y P Tu, Y.P ; Holmes, J.H. Fragmentation of Substituted Oxonium Ions: The Role of Ion-Neutral Complexes J. Am. Soc. Mass Spectro. 1999, 10, 386-392.

3. Lesthaeghe, D.; Speybroeck, V.V.; Marin, G.E.; Waroquier, M. The Rise and Fall of Direct Mechanism in Methanol-to-Olefin Catalysis : An Overview of Theoretical Contributions. Ind. Eng. Chem. Res. 2007, 46, 8832-8838

4. CHEMKIN a chemical kinetic program originally published in 1982 by R.J. Kee and J.A. Miller at Sandia National Labs. Livermore CA; currently developed and marketed by Reaction Design, San-Diego, CA.

5. Goodwin D. CANTERA. A free chemical kinetic software for solving chemical reacting laminar flows. Developed at California Institute of Technology. Available at http://sourceforge.net/projects/cantera/

6. Benson S.W. Thermochemical Kinetics, Wiley, N.Y. 1976.

7. Chase, M.W, Jr.; Davies, C.A; Downey, J.R. Jr.; Frurip, D.J.; McDonald, R.A.; Syverud, A.N. The JANAF Tables, 3rd edition, J. Phys. Chem. Ref. Data 1985, 14, Suppl. No. 1. 
8. Wagman, D.D.; Evans, W.H.; Parker, V.S.; Schumm, R.H.; Holow, I.; Bailey, S.M.; Churney, K.L.; Nuttall, R.L. The NBS Tables of Chemical Thermodynamic Properties Selected Values for Inorganic and $\mathrm{C}_{1}-\mathrm{C}_{2}$ Organic substances J. Phys. Chem. Ref. Data 1982, 11 Suppl 2.

9. Gurvich V.N. Veyts I.V. and Alcock C.B. "Thermodynamic Properties of Individual Substances" $4^{\text {th }}$ edition Hemisphere N.Y.1992.

10. Lias, S.G.; Bartmess, J.E.; Liebman, J.F.; Holmes, J.L.; Levin, R.D.; Mallard, W.G. Gas-Phase Ion and Neutral Thermochemistry, J. Phys. Chem. Ref. Data, 1988, 17, Suppl. 1.

11. Afeefy, H.Y.; Liebmann, J.F \& Stein, S.E. NIST Standard Reference Database Number 69 Edited by W.G Mallard W.G. \& Linstrom, P.J. (National Institute of Standards and Technology). <http://webbook.nist.gov/chemistry>

12. Baboul, A.G.; Curtis, L.A.; Redfem, P.C.; Raghavachari K. Gaussian-3 theory using density functional geometries and zero-point energies J. Chem Phys, 1999, 110, 7650.

13. Gaussian 03, Revision B.02,

M. J. Frisch, G. W. Trucks, H. B. Schlegel, G. E. Scuseria, M. A. Robb, J. R. Cheeseman, J. A. Montgomery, Jr., T. Vreven, K. N. Kudin, J. C. Burant, J. M. Millam, S. S. Iyengar, J. Tomasi, V. Barone, B. Mennucci, M. Cossi, G. Scalmani, N. Rega, G. A. Petersson, H. Nakatsuji, M. Hada, M. Ehara, K. Toyota, R. Fukuda, J. Hasegawa, M. Ishida, T. Nakajima, Y. Honda, O. Kitao, H. Nakai, M. Klene, X. Li, J. E. Knox, H. P. Hratchian, J. B. Cross, C. Adamo, J. Jaramillo, R. Gomperts, R. E. Stratmann, O. Yazyev, A. J. Austin, R. Cammi, C. Pomelli, J. W. Ochterski, P. Y. Ayala, K. Morokuma, G. A. Voth, P. Salvador, J. J. Dannenberg, V. G. Zakrzewski, S. Dapprich, A. D. Daniels, M. C. Strain, O. Farkas, D. K. Malick, A. D. Rabuck, K. Raghavachari, J. B. Foresman, J. V. Ortiz, Q. Cui, A. G. Baboul, S. Clifford, J. Cioslowski, B. B. Stefanov, G. Liu, A. Liashenko, P. Piskorz, I. Komaromi, R. L. Martin, D. J. Fox, T. Keith, M. A. Al-Laham, C. Y. Peng, A. Nanayakkara, M. Challacombe, P. M. W. Gill, B. 
Johnson, W. Chen, M. W. Wong, C. Gonzalez, and J. A. Pople, Gaussian, Inc., Pittsburgh PA, 2003.

14. Chem3D is a "CambrigeSoft" program for molecular modeling and display with semi-empirical calculations built in.

15. McBride B.J.; and Gordon, S. Computer Program for Calculating and Fitting Thermodynamic Functions. NASA RP 12711992.

16. Ruscic B. Active Thermochemical Tables (ATcT) $2013<$ http://atct.anl.gov>

17. Brown, R.C.; Eraslan, A.N. Simulation of ionic structure in lean and close-tostoichiometric acetylene flames Comb Flame 1988, 73,1-21.

18. "Computational Chemistry Comparison and Benchmark Data Base " (CCCBDB) Release 16a, August 2013, NIST Standard Reference Database 101

19. Lias, S.G.; Liebman, J.F.; Levin, R.D. Heats of Formation of Protonated Molecules J. Phys. Chem. Ref. Data 1984, 13, 695-808.

20. Goos, E.; Burcat, A.; Ruscic, B. Extended Third Millennium Ideal Gas and Condensed Phase Thermochemical Database for Combustion with Updates from Active Thermochemical Tables" < http://burcat.technion.ac.il/dir $>$

21. NIST CCCBDB (Computational Chemistry Comparison Benchmark) available at $<$ cccbdb.nist.gov $>$.

22. Bakowies D. Ab Initio Thermochemistry with High-Level Isodesmic Corrections: Validation of the ATOMIC Protocol for a Large Set of Compounds with First-Row Atoms (H, C, N, O, F) J. Phys. Chem. A, 2009 113, 11517-11534 
23. Simmie, J.; Sommers, K. Benchmarking Compound Methods (CBS-QB3, CBSAPNO, G3, G4, W1BD) against the Active Thermochemical Tables: A Litmus Test for Cost-Effective Molecular Formation Enthalpies J. Phys. Chem. A, 2015 119(28), 72357246 .

$$
\begin{gathered}
\Delta f(\mathrm{~T}) \mathrm{H}_{\mathrm{T}} \mathrm{S}_{\mathrm{T}} \Delta_{f} \mathrm{G}(\mathrm{T}) \\
\text { for } \\
\mathrm{CH}-O H_{2}+, \mathrm{CH}_{2}-\mathrm{OH}_{2}+, \mathrm{CH}_{3}-\mathrm{OH}_{2}+, \mathrm{CH}_{3} \mathrm{O}=\mathrm{CH}_{2}+, \\
{\left[\mathrm{CH}_{2}-\mathrm{CH}_{2}\right] \mathrm{OH}+, \mathrm{CH}_{3} \mathrm{OH}-\mathrm{CH}_{2}+,\left(\mathrm{CH}_{3}\right)_{2} \mathrm{OH}+,} \\
\left(\mathrm{CH}_{3}\right)_{3} \mathrm{O}+, \mathrm{n}-\mathrm{C}_{3} \mathrm{H}_{7} \mathrm{OH}_{2}+,\left(\mathrm{CH}_{3}\right)_{2} \mathrm{CHOH}_{2}^{+}, \\
\left(\mathrm{CH}_{3}\right)_{2} \mathrm{OCH}_{2}+, \mathrm{CH}_{3}\left[\mathrm{CH}_{-} \mathrm{CH}_{2}\right] \mathrm{OH}+ \\
{\left[\mathrm{CH}_{2}-\mathrm{CH}_{2}-\mathrm{CH}_{2}\right] \mathrm{OH}+,\left(\mathrm{CH}_{3}\right)_{2} \mathrm{OC} \mathrm{H}_{5}+,} \\
{\left[\mathrm{CH}_{2}-\mathrm{CH}_{2}-\mathrm{CH}_{2}-\mathrm{CH}_{2}\right] \mathrm{OH}+, \mathrm{C}_{2} \mathrm{H}_{5} \mathrm{OH}_{2}+,\left(\mathrm{C}_{2} \mathrm{H}_{5}\right)_{2} \mathrm{OH}+,} \\
\left(\mathrm{C}_{2} \mathrm{H}_{5}\right)_{2} \mathrm{OCH}_{3}+,\left[\mathrm{CH}_{2}-\mathrm{CH}_{2}-\mathrm{CH}_{2}-\mathrm{CH}_{2}-\mathrm{CH}_{2}\right] \mathrm{OH}+ \\
\left(\mathrm{C}_{2} \mathrm{H}_{5}\right)_{3} \mathrm{O}+
\end{gathered}
$$




\title{
Supporting Information \\ The Ideal Gas Thermochemistry of Oxonium Cations
}

\author{
Alexander Burcat*广 Elke Goos ${ }^{\ddagger}$ \\ ${ }^{\dagger}$ Faculty of Aerospace Engineering, Technion-IIT, Haifa, 32000, Israel \\ ${ }^{*}$ Institute of Combustion Technology, DLR (German Aerospace Center), \\ Stuttgart, 70569 Germany.
}

\section{* corresponding author. e-mail: aer0201@ technion.ac.il}

\begin{abstract}
Table S1 7 term NASA polynomials of oxonium compounds (thermal electron convention) of protonated compounds and radical cations.

CH3O+ Methylidene Oxonium cation $\mathrm{CHOH}_{2}+\mathrm{HF} 298=1042.05 \pm 8$. $\mathrm{kJ} / \mathrm{mol}$ Burcat G3B3 CHOH2+ Oxonium T $5 / 15 \mathrm{C} \quad 1 . \mathrm{H} \quad 3.0 \quad 1 . \mathrm{E}-1 . \mathrm{G} \quad 298.150 \quad 6000.0001000$.

$4.39380166 \mathrm{E}+00 \quad 7.16728474 \mathrm{E}-03-2.46127134 \mathrm{E}-06 \quad 3.85124272 \mathrm{E}-10-2.25476964 \mathrm{E}-14$

$1.23503552 \mathrm{E}+05 \quad 3.25046236 \mathrm{E}-01 \quad 1.69308260 \mathrm{E}+00 \quad 1.45930724 \mathrm{E}-02-9.09655507 \mathrm{E}-06$

$\begin{array}{lllll}1.76040858 \mathrm{E}-09 & 5.12383125 \mathrm{E}-13 & 1.24252331 \mathrm{E}+05 & 1.42806460 \mathrm{E}+01 & 1.25329093 \mathrm{E}+05\end{array}$

$\mathrm{CH} 4 \mathrm{O}^{*}+$ Methylene Oxonium cation $\mathrm{CH}_{2} \mathrm{OH}_{2}{ }^{*}+\mathrm{HF} 298=825.9 \pm 4.5 \mathrm{~kJ} / \mathrm{mol}$ Ruscic ATCT 2013

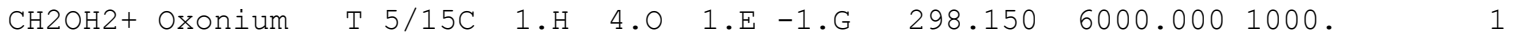
$5.04696028 \mathrm{E}+00 \quad 8.83268034 \mathrm{E}-03-2.96312821 \mathrm{E}-06 \quad 4.55893988 \mathrm{E}-10-2.63627238 \mathrm{E}-14 \quad 2$

$9.72441646 \mathrm{E}+04-1.99964848 \mathrm{E}+00 \quad 1.59132994 \mathrm{E}+00 \quad 2.00143834 \mathrm{E}-02-1.60986921 \mathrm{E}-05 \quad 3$

$6.60975763 \mathrm{E}-09-7.70735214 \mathrm{E}-13 \quad 9.80978732 \mathrm{E}+04 \quad 1.53918843 \mathrm{E}+01 \quad 9.93323720 \mathrm{E}+04 \quad 4$
\end{abstract}

CH5O+ Methyl Oxonium (Rydberg cation) $\mathrm{CH}_{3} \mathrm{OH}_{2}+\mathrm{HF} 298=583.7 \pm 8$. kJ Burcat G3B3 $\mathrm{CH} 5 \mathrm{O}+\mathrm{CH} 3 \mathrm{OH} 2+\mathrm{T} 11 / 14 \mathrm{C} \quad 1 . \mathrm{H} \quad 5.0 \quad 1 . \mathrm{E}-1 . \mathrm{G} \quad 298.150 \quad 6000.0001000$. $4.12018251 \mathrm{E}+00 \quad 1.23179143 \mathrm{E}-02-4.21315216 \mathrm{E}-06 \quad 6.57462537 \mathrm{E}-10-3.84191498 \mathrm{E}-14$ $6.81422088 \mathrm{E}+04 \quad 1.71193370 \mathrm{E}+00 \quad 2.63252920 \mathrm{E}+00 \quad 9.23812483 \mathrm{E}-03 \quad 1.43800480 \mathrm{E}-05$

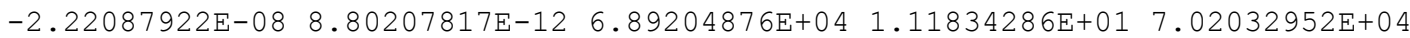

C2H5O+ Methyl Methylene Oxonium $\mathrm{CH}_{3}-\mathrm{O}=\mathrm{CH}_{2}+\mathrm{HF} 298=676.9 \pm 8$. kJ Burcat G3B3 $\mathrm{C} 2 \mathrm{H} 5 \mathrm{O}+\mathrm{CH} 3-\mathrm{O}=\mathrm{CH} 2+\mathrm{T} 11 / 14 \mathrm{C} \quad 2 . \mathrm{H} \quad 5.0 \quad 1 . \mathrm{E}-1 . \mathrm{G} \quad 298.150 \quad 6000.0001000$.

$5.23682452 \mathrm{E}+001.45674929 \mathrm{E}-02-5.15192045 \mathrm{E}-06 \quad 8.22867349 \mathrm{E}-10-4.88896791 \mathrm{E}-14$ $7.87093166 \mathrm{E}+04-3.31148010 \mathrm{E}+00 \quad 3.32715925 \mathrm{E}+00 \quad 7.43454980 \mathrm{E}-03 \quad 2.71006098 \mathrm{E}-05$

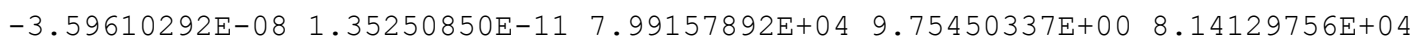

C2H5O+ Protonated Oxyrane $\left[\mathrm{CH}_{2}-\mathrm{CH}_{2}\right] \mathrm{OH}+\mathrm{HF} 298=715.07 \pm 0.62 \mathrm{~kJ}$ Ruscic ATCT D 2013 $\mathrm{C} 2 \mathrm{H} 5 \mathrm{O}+(\mathrm{CH} 2 \mathrm{CH} 2) \mathrm{OH}+\mathrm{T} 12 / 14 \mathrm{C} \quad 2 . \mathrm{H} \quad 5 . \mathrm{O} \quad 1 . \mathrm{E}-1 . \mathrm{G} \quad 298.150 \quad 6000.0001000$. $5.70791482 \mathrm{E}+001.39321006 \mathrm{E}-02-4.87755699 \mathrm{E}-06 \quad 7.73937429 \mathrm{E}-10-4.57782079 \mathrm{E}-14$ $8.30274882 \mathrm{E}+04-8.39211861 \mathrm{E}+00-5.22964914 \mathrm{E}-01 \quad 2.30971327 \mathrm{E}-02 \quad 5.20358577 \mathrm{E}-06$ $\begin{array}{llll}-2.33955821 \mathrm{E}-08 & 1.11084462 \mathrm{E}-11 & 8.51270059 \mathrm{E}+04 & 2.57116207 \mathrm{E}+01 \quad 8.60026628 \mathrm{E}+04\end{array}$

$\mathrm{C} 2 \mathrm{H} 6 \mathrm{O}^{*}+$ Methyl Methylene Proton Oxonium $\mathrm{CH}_{3}-\mathrm{OH}-\mathrm{CH}_{2}{ }^{*}+\mathrm{HF} 298=797.6 \pm 8$. kJ Burcat $\mathrm{C} 2 \mathrm{H} 6 \mathrm{O}+\mathrm{CH} 3-\mathrm{OH}-\mathrm{CH} 2+\mathrm{T} \quad 5 / 15 \mathrm{C} \quad 2 . \mathrm{H} \quad 6.0 \quad 1 . \mathrm{E}-1 . \mathrm{G} \quad 298.150 \quad 6000.000 \quad 1000$. $6.85380737 \mathrm{E}+001.52932218 \mathrm{E}-02-5.29722518 \mathrm{E}-06 \quad 8.34043510 \mathrm{E}-10-4.90531913 \mathrm{E}-14$

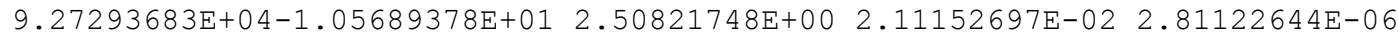

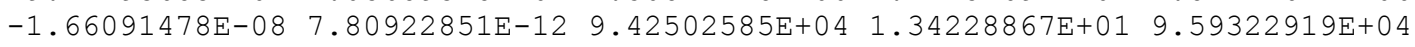

$\mathrm{C} 2 \mathrm{H} 7 \mathrm{O}+$ DiMethyl Oxonium $\left(\mathrm{CH}_{3}\right)_{2}-\mathrm{OH}+\mathrm{HF} 298=562.24 \pm 8$. $\mathrm{kJ}$ Burcat G3B3 $\mathrm{C} 2 \mathrm{H} 7 \mathrm{O}+(\mathrm{CH} 3) 2 \mathrm{OH}+\mathrm{T} 11 / 14 \mathrm{C} \quad 2 . \mathrm{H} \quad 7 . \mathrm{O} \quad 1 . \mathrm{E}-1 . \mathrm{G} \quad 298.150 \quad 6000.000 \quad 1000$. $5.81266575 \mathrm{E}+001.89420633 \mathrm{E}-02-6.61986229 \mathrm{E}-061.04878304 \mathrm{E}-09-6.19569490 \mathrm{E}-14$ $6.44582085 \mathrm{E}+04-7.03041901 \mathrm{E}+00 \quad 3.49195920 \mathrm{E}+00 \quad 9.77638270 \mathrm{E}-03 \quad 3.50079732 \mathrm{E}-05$ $\begin{array}{llllll}-4.70880441 \mathrm{E}-08 & 1.79334219 \mathrm{E}-11 & 6.59209412 \mathrm{E}+04 & 8.89898119 \mathrm{E}+00 & 6.76213030 \mathrm{E}+04\end{array}$

C2H7O+ Ethyl Oxonium $\mathrm{C}_{2} \mathrm{H}_{5} \mathrm{OH}_{2}+\mathrm{HF} 298=527.76 \pm 8$. kJ Burcat G3B3

$\mathrm{C} 2 \mathrm{H} 7 \mathrm{O}+\mathrm{C} 2 \mathrm{H} 5 \mathrm{OH} 2+\mathrm{T} 11 / 14 \mathrm{C} \quad 2 . \mathrm{H} \quad 7.0 \quad 1 . \mathrm{E}-1 . \mathrm{G} \quad 298.150 \quad 6000.0001000$. 
$6.18891803 \mathrm{E}+00 \quad 1.79926340 \mathrm{E}-02-6.24009542 \mathrm{E}-06 \quad 9.83369694 \mathrm{E}-10-5.78725674 \mathrm{E}-14$

$6.03219647 \mathrm{E}+04-7.85592076 \mathrm{E}+00 \quad 2.86917130 \mathrm{E}+00 \quad 1.73908141 \mathrm{E}-02 \quad 1.49889555 \mathrm{E}-05$

$\begin{array}{llllll}-2.77297126 \mathrm{E}-08 & 1.13477254 \mathrm{E}-11 & 6.17634220 \mathrm{E}+04 & 1.17829996 \mathrm{E}+01 & 6.34748169 \mathrm{E}+04\end{array}$

C3H7O+ PropyleneOxide Oxonium $\mathrm{CH}_{3}\left[\mathrm{CH}-\mathrm{CH}_{2}\right] \mathrm{OH}+\mathrm{HF} 298=648.0 \pm 8$. kJ Burcat G3B3 C3H7O+ MeOxoProt T12/14C 3.H 7.O 1.E -1.G $298.150 \quad 6000.0001000$.

$8.00791650 \mathrm{E}+001.98766698 \mathrm{E}-02-7.00852891 \mathrm{E}-061.11739544 \mathrm{E}-09-6.63134336 \mathrm{E}-14$

$7.37876008 \mathrm{E}+04-1.91729839 \mathrm{E}+01 \quad 1.71414238 \mathrm{E}-01 \quad 3.05635474 \mathrm{E}-02 \quad 6.92713138 \mathrm{E}-06$

$\begin{array}{llllll}-2.93671651 \mathrm{E}-08 & 1.36322114 \mathrm{E}-11 & 7.65168794 \mathrm{E}+04 & 2.40418371 \mathrm{E}+01 & 7.79360419 \mathrm{E}+04\end{array}$

$\mathrm{C} 3 \mathrm{H} 7 \mathrm{O}+$ CycloTriMethyleneoxide Oxonium $\left[\mathrm{CH}_{2} \mathrm{CH}_{2} \mathrm{CH}_{2}\right] \mathrm{OH}+\mathrm{HF} 298=640.85 \pm 8$. kJ Burcat G3B3 C3H70+ CyOxonium T12/14C 3.H $7.0 \quad 1 . \mathrm{E}-1 . \mathrm{G} \quad 298.150 \quad 6000.0001000$.

$7.43398460 \mathrm{E}+00 \quad 2.04359385 \mathrm{E}-02-7.22191223 \mathrm{E}-06 \quad 1.15327670 \mathrm{E}-09-6.85237610 \mathrm{E}-14$

$7.29613893 \mathrm{E}+04-1.78357142 \mathrm{E}+01-1.51555883 \mathrm{E}+00 \quad 3.27175286 \mathrm{E}-02 \quad 8.98977573 \mathrm{E}-06$

$-3.44443374 \mathrm{E}-08 \quad 1.59853557 \mathrm{E}-11 \quad 7.60548693 \mathrm{E}+04 \quad 3.14505091 \mathrm{E}+01 \quad 7.70760995 \mathrm{E}+04$

C3H8O*+ Dimethyl Methylene Oxonium $\left(\mathrm{CH}_{3}\right)_{2} \mathrm{O}-\mathrm{CH}_{2}{ }^{*}+\mathrm{HF} 298=772.8 \pm 8$. kJ Burcat G3B3 $\mathrm{C} 3 \mathrm{H} 8 \mathrm{O}+(\mathrm{CH} 3) 2 \mathrm{O}=\mathrm{CH} 2 \mathrm{~T} 10 / 14 \mathrm{C} \quad 3 . \mathrm{H} \quad 8.0 \quad 1 . \mathrm{E}-1 . \mathrm{G} \quad 298.150 \quad 6000.0001000$.

$8.67839202 \mathrm{E}+00 \quad 2.17590005 \mathrm{E}-02-7.63698179 \mathrm{E}-06 \quad 1.21342751 \mathrm{E}-09-7.18282028 \mathrm{E}-14$

$8.86610510 \mathrm{E}+04-1.98461405 \mathrm{E}+01 \quad 3.64462504 \mathrm{E}+00 \quad 2.27457201 \mathrm{E}-02 \quad 1.85409505 \mathrm{E}-05$

$\begin{array}{lllll}-3.57734005 \mathrm{E}-08 & 1.47841149 \mathrm{E}-11 & 9.07509991 \mathrm{E}+04 & 9.46516110 \mathrm{E}+00 & 9.29487126 \mathrm{E}+04\end{array}$

C3H9O+ Trimethyl Oxonium $\left(\mathrm{CH}_{3}\right)_{3} \mathrm{O}+\mathrm{HF} 298=541.716 \pm 8$. $\mathrm{kJ} / \mathrm{mol}$ Burcat G3B3

$\mathrm{C} 3 \mathrm{H} 90+(\mathrm{CH} 3) 30+\mathrm{T} 10 / 14 \mathrm{C} \quad 3 . \mathrm{H} \quad 9.0 \quad 1 . \mathrm{E}-1 . \mathrm{G} \quad 298.150 \quad 6000.000 \quad 1000$.

$7.59728406 \mathrm{E}+00 \quad 2.54784347 \mathrm{E}-02-8.99285110 \mathrm{E}-06 \quad 1.43435890 \mathrm{E}-09-8.51363342 \mathrm{E}-14$

$6.09096282 \mathrm{E}+04-1.64610245 \mathrm{E}+01 \quad 4.51970904 \mathrm{E}+001.18541575 \mathrm{E}-02 \quad 4.97969882 \mathrm{E}-05$

$\begin{array}{llllll}-6.52816266 \mathrm{E}-08 & 2.45428621 \mathrm{E}-11 & 6.29561255 \mathrm{E}+04 & 5.10913040 \mathrm{E}+00 & 6.51530878 \mathrm{E}+04\end{array}$

1

C3H9O+ n-Propanol Protonated Oxonium n-C3H7OH2+ HF298=501.85 \pm 8 . kJ Burcat G3B3

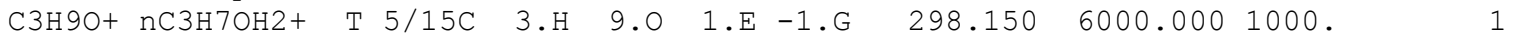

$9.44845876 \mathrm{E}+00 \quad 2.35494635 \mathrm{E}-02-8.48735271 \mathrm{E}-06 \quad 1.36974058 \mathrm{E}-09-8.18959901 \mathrm{E}-14$

$5.54563517 \mathrm{E}+04-2.49192675 \mathrm{E}+01 \quad 1.57568968 \mathrm{E}+00 \quad 2.98650264 \mathrm{E}-02 \quad 1.75864292 \mathrm{E}-05$ $\begin{array}{llllll}-4.05058476 \mathrm{E}-08 & 1.72771164 \mathrm{E}-11 & 5.84771733 \mathrm{E}+04 & 1.97302170 \mathrm{E}+01 & 6.03578563 \mathrm{E}+04\end{array}$

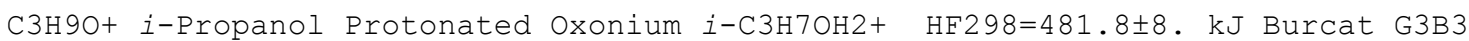
$\mathrm{C} 3 \mathrm{H} 9 \mathrm{O}+\mathrm{iC} 3 \mathrm{H} 7 \mathrm{OH} 2+\mathrm{T} 5 / 15 \mathrm{C} \quad 3 . \mathrm{H} \quad 9.0 \quad 1 . \mathrm{E}-1 . \mathrm{G} \quad 298.150 \quad 6000.000 \quad 1000$.

$9.57409469 \mathrm{E}+00 \quad 2.31713068 \mathrm{E}-02-8.17316606 \mathrm{E}-06 \quad 1.29960391 \mathrm{E}-09-7.68969622 \mathrm{E}-14$

$5.19914246 \mathrm{E}+04-2.52943675 \mathrm{E}+01 \quad 2.25481526 \mathrm{E}+00 \quad 3.15666564 \mathrm{E}-02 \quad 9.08108465 \mathrm{E}-06$

$-3.10362905 \mathrm{E}-08 \quad 1.39286765 \mathrm{E}-11 \quad 5.46444712 \mathrm{E}+04 \quad 1.55201868 \mathrm{E}+01 \quad 5.67452562 \mathrm{E}+04$

C4H9O+ TetraHydroFuran Oxonium $\left[\mathrm{CH}_{2} \mathrm{CH}_{2} \mathrm{CH}_{2} \mathrm{CH}_{2}\right] \mathrm{OH}+\mathrm{HF} 298=529.07 \pm 8$. kJ Burcat G3B3 $\mathrm{C} 4 \mathrm{H} 90+$ TetHydFuranT12/14C $\quad 4 . \mathrm{H} \quad 9.0 \quad 1 . \mathrm{E}-1 . \mathrm{G} \quad 298.150 \quad 6000.0001000$. $9.27420638 \mathrm{E}+00 \quad 2.68100376 \mathrm{E}-02-9.51325112 \mathrm{E}-06 \quad 1.52337781 \mathrm{E}-09-9.06884102 \mathrm{E}-14$

$5.83840237 \mathrm{E}+04-2.82047764 \mathrm{E}+01-2.08366085 \mathrm{E}+00 \quad 4.20226784 \mathrm{E}-021.12339571 \mathrm{E}-05$ $\begin{array}{lllll}-4.29010524 \mathrm{E}-08 & 1.97317600 \mathrm{E}-11 & 6.23614523 \mathrm{E}+04 & 3.45188773 \mathrm{E}+01 & 6.36317714 \mathrm{E}+04\end{array}$

C4H11O+ Ethyl DiMethyl Oxonium $\mathrm{C}_{2} \mathrm{H}_{5}-\mathrm{O}-\left(\mathrm{CH}_{3}\right)_{2}+\mathrm{HF} 298=498 . \pm 8$. kJ Burcat G3B3 C4H110+ Oxonium T10/14C 4.H 11.O $1 . \mathrm{E}-1 . \mathrm{G} \quad 298.150 \quad 6000.0001000$. $9.41866464 \mathrm{E}+00 \quad 3.13815691 \mathrm{E}-02-1.11039894 \mathrm{E}-05 \quad 1.77410334 \mathrm{E}-09-1.05428724 \mathrm{E}-13$ $5.46000657 \mathrm{E}+04-2.53557791 \mathrm{E}+01 \quad 4.76501693 \mathrm{E}+00 \quad 1.78290766 \mathrm{E}-02 \quad 5.63814406 \mathrm{E}-05$ $\begin{array}{lllll}-7.68147655 \mathrm{E}-08 & 2.92041503 \mathrm{E}-11 & 5.73201177 \mathrm{E}+04 & 5.46915280 \mathrm{E}+00 & 5.98933671 \mathrm{E}+04\end{array}$

C4H11O+ DiEthyl Oxonium $\left(\mathrm{C}_{2} \mathrm{H}_{5}\right)_{2} \mathrm{OH}+\mathrm{HF} 298=468.08 \pm 8$. kJ/mol Burcat G3B3 $\mathrm{C} 4 \mathrm{H} 11 \mathrm{O}+(\mathrm{C} 2 \mathrm{H} 5) 2 \mathrm{OH}+\mathrm{T} 11 / 14 \mathrm{C} \quad 4 . \mathrm{H} \quad 11 . \mathrm{O} \quad 1 . \mathrm{E}-1 . \mathrm{G} \quad 298.150 \quad 6000.0001000$.

$9.93034521 \mathrm{E}+00 \quad 3.02853390 \mathrm{E}-02-1.06660779 \mathrm{E}-05 \quad 1.69877827 \mathrm{E}-09-1.00731034 \mathrm{E}-13$

$5.09606767 \mathrm{E}+04-2.83500513 \mathrm{E}+01 \quad 3.23373030 \mathrm{E}+00 \quad 3.04473021 \mathrm{E}-02 \quad 2.67898693 \mathrm{E}-05$

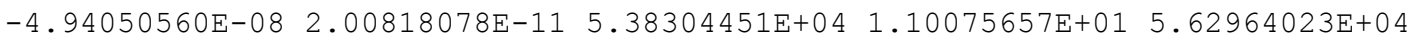

C5H110+ PentaHydroxyPyran Oxonium $\left[\mathrm{CH}_{2} \mathrm{CH}_{2} \mathrm{CH}_{2} \mathrm{CH}_{2} \mathrm{CH}_{2}\right] \mathrm{OH}+\mathrm{HF} 298=510.96 \pm 8$. kJ Burcat G3B3

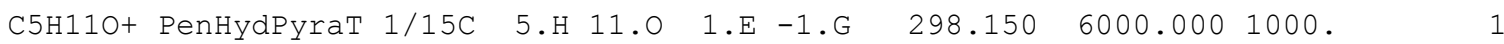
$1.10492507 \mathrm{E}+01 \quad 3.32787293 \mathrm{E}-02-1.18463934 \mathrm{E}-05 \quad 1.90101634 \mathrm{E}-09-1.13335364 \mathrm{E}-13 \quad 2$ 
C5H130+ Methyl DiEthyl Oxonium $\mathrm{CH}_{3}-\mathrm{O}-\left(\mathrm{C}_{2} \mathrm{H}_{5}\right)_{2}+\mathrm{HF} 298=455.7 \pm 8$. kJ Burcat G3B3

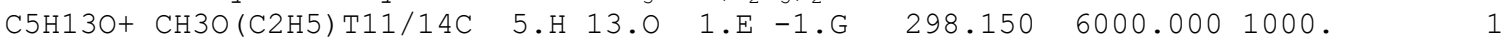

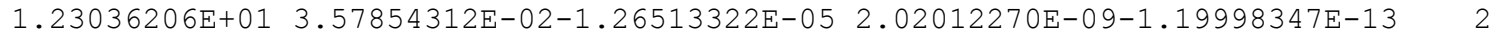
$\begin{array}{llllll}4.82194810 \mathrm{E}+04-4.14071543 \mathrm{E}+01 & 2.26691809 \mathrm{E}+00 & 4.31421706 \mathrm{E}-02 & 2.28259869 \mathrm{E}-05 & 3\end{array}$ $\begin{array}{lllllll}-5.39722310 \mathrm{E}-08 & 2.30749993 \mathrm{E}-11 & 5.21111296 \mathrm{E}+04 & 1.56938999 \mathrm{E}+01 & 5.48104458 \mathrm{E}+04 & 4\end{array}$

C6H15O+ TriEthyl Oxonium $\left(\mathrm{C}_{2} \mathrm{H}_{5}\right){ }_{3} \mathrm{O}+\mathrm{HF} 298=414.7 \pm 8$. $\mathrm{kJ} / \mathrm{mol}$ Burcat G3B3 $\mathrm{C} 6 \mathrm{H} 15 \mathrm{O}+(\mathrm{C} 2 \mathrm{H} 5) 3 \mathrm{O}+\mathrm{T} 11 / 14 \mathrm{C} \quad 6 . \mathrm{H} \quad 15 . \mathrm{O} \quad 1 . \mathrm{E}-1 . \mathrm{G} \quad 298.150 \quad 6000.000 \quad 1000$. $\begin{array}{lll}1.38748821 \mathrm{E}+01 & 4.23805960 \mathrm{E}-02-1.50162400 \mathrm{E}-05 & 2.40134262 \mathrm{E}-09-1.42793078 \mathrm{E}-13\end{array}$ $4.23572955 \mathrm{E}+04-5.00923764 \mathrm{E}+01 \quad 3.65827166 \mathrm{E}+00 \quad 4.63430467 \mathrm{E}-02 \quad 2.98234566 \mathrm{E}-05$ $\begin{array}{lllll}-6.17841058 \mathrm{E}-08 & 2.54571186 \mathrm{E}-11 & 4.65724949 \mathrm{E}+04 & 9.09434099 \mathrm{E}+00 & 4.98764209 \mathrm{E}+04\end{array}$

* Oxoniums that are radical cations or distonic ions. 